\title{
Quantitative Analysis of COVID-19 Pandemic Responses Based on an Improved SEIR-SD Model
}

\author{
Yang Liu, ${ }^{1}$ Bingrui Liu, ${ }^{2}$ Yi Deng, ${ }^{2}$ and Jia Liu $\mathbb{D}^{2}$ \\ ${ }^{1}$ School of Management, Wuhan University of Technology, Wuhan, China \\ ${ }^{2}$ School of Information and Safety Engineering, Zhongnan University of Economics and Law, Wuhan, China \\ Correspondence should be addressed to Jia Liu; whutrobin@163.com
}

Received 17 May 2021; Revised 6 August 2021; Accepted 3 January 2022; Published 1 February 2022

Academic Editor: Chenquan Gan

Copyright (c) 2022 Yang Liu et al. This is an open access article distributed under the Creative Commons Attribution License, which permits unrestricted use, distribution, and reproduction in any medium, provided the original work is properly cited.

In late 2019, the COVID-19 pandemic began to spread over the world, causing millions of deaths. In the first few months of the pandemic, several countries (such as China) prevented the spread of the pandemic successfully. By contrast, the pandemic in many other countries was not controlled well. For example, India encountered a second serious outbreak of COVID-19 from April 2021 due to the poor resistance measures implemented by the government. To figure out the effective countermeasures to the pandemic, this research proposes a COVID-19 pandemic and its response system, which consists of the infection subsystem, the quarantine subsystem, and the medical subsystem. On this basis, an improved SEIR-SD model is established which is utilized to analyze the response measures to the pandemic quantitatively. This model successfully simulates the actual epidemic scenarios in Wuhan, which verifies its effectiveness. Afterward, the impact of hospital administration rate, quarantine rate, average contact number, and contact infection rate on the cumulative number of infections and deaths are analyzed by simulation. The results show that both the medical and administrative efforts, especially in the early stage of the epidemic, are significant in reducing the number of infections and shortening the epidemic period. In the medical aspect, the more stringent quarantine brings the earlier inflection point of the epidemic; more importantly, improving the treatment rate significantly reduces the scale of the epidemic. In the administrative aspect, enforcing individual protection and strict community closure can effectively cut off the transmission of the virus and curb the spread of the epidemic. Finally, this research proposes several practical suggestions in response to the COVID19 pandemic. The main contribution of this research is that the effects of different response measures on the number of new infections daily and the cumulative number of deaths of a country or region in the COVID-19 pandemic are estimated quantitatively based on modeling and simulation.

\section{Introduction}

The COVID-19 epidemic was discovered in Wuhan in late 2019 and spread over the world. After the outbreak, the Chinese national government and Wuhan local government immediately adopted a series of measures to control the spread of the virus. These measures include but are not limited to closing communities, enforcing individual protection, isolating suspected cases, and establishing temporary treatment centers for mildly infected cases. Simultaneously, medical staff and supplies from all over China supported Wuhan rapidly, which greatly reduced the death rate of confirmed cases in Wuhan. However, the pandemic in many other countries was not controlled well.
For example, India encountered a second serious outbreak of COVID-19 from April 2021 due to the poor resistance measures implemented by the government.

From the perspective of system science, the epidemic of infectious diseases in the population is a complex diffusion process. The analysis and prediction of infectious disease spread based on models can help understand the epidemic mechanism and the inherent laws and provide a theoretical basis for the choice of intervention measures. The models are established based on either the micro- or macroperspectives. The micromodels focus on the individuals in the crowd. There is a contact network with individuals. The contact between the infected cases and the suspected ones leads to the state change of the suspected ones, forming the 
transmission dynamics process on the network [1]. There are two main research fields of network-based micromodel: the first one is to study the spread of infectious diseases in ideal networks, such as small-world networks and scale-free networks, which partially depict the characteristics of real social networks [2]. The second one is to study the spread of infectious diseases on the real network. The basic method is to carry out actual investigations and construct a contact network close to the real one [3]. Macroscopic modeling regards the population as a whole and focuses on the changes of its state. The composite population method considers the spatial heterogeneity of the population and divides the population into different groups, which are coupled by the flow of people, forming a complex dynamic system [4]. Correspondingly, the single group method shows the epidemic process of infectious diseases in the number of susceptible, infectious, and other types of people. The most popular single population model is the compartment model. One of the classic compartment models is the SIR model, which was proposed by Kermack et al. in 1927 [5]. Based on the SIR model, the SIS model [6], SIRS model [7], and SEIR model [8] are developed. The SEIR model is one of the most representative mathematical models of infectious disease dynamics in a fixed population. The model considers the incubation period of infectious diseases and is suitable for large-scale modeling of the COVID-19 epidemic on the country and city level. Therefore, this paper intends to analyze the COVID-19 epidemic by the improvement of this model.

The system dynamics model [9] was first proposed in 1956 by Forrester of the Massachusetts Institute of Technology. It has been widely used in industry, agriculture, economy, management, medicine, transportation, ecology, environment, energy, military, and many other fields. For example, Assuncao et al. used system dynamics to establish an urban sustainable development system considering the natural factors, physiological feelings, and psychological feelings [10]. Ekinci et al. utilized the model to assess the future impact of various air quality factors on environmental sustainability [11]. As one of the most powerful tools to study complex systems, system dynamics has a broad prospect on public health, in which the combination of system dynamics and the classical models of infectious diseases is a new field of infectious disease research $[12,13]$. $\mathrm{Li}$ et al. combined the system dynamics with a multicompartment model to predict the development trend of the COVID-19 epidemic [14]. In this study, the system dynamics model is combined with the SEIR model so that the solution process of the SEIR model can be simulated and visualized. More importantly, the effect of different variables on simulation results can be analyzed.

After the outbreak of COVID-19, many scholars engaged in the study of the epidemic by establishing mathematical models. Tang and Zhao used the classical SEIR model to fit the epidemic development trend [15, 16]. Considering quarantine measures and types of the infected population, Wei et al. proposed the SEIR + CAQ model to predict the number of infected cases [17]. In the early stage of COVID19 , many studies focused on the scale prediction of the epidemic. For example, British scholar Jonathan et al. predicted in January 2020 that infected cases in Wuhan would reach 190,000 on February $4^{\text {th }}$ [18]. Shen et al. estimated the basic and effective reproduction times of COVID-19 [19]. They predicted the peak time and scale of the epidemic based on the existing epidemiological data and the dynamic model. They also estimated that the number of infected people would ultimately be less than 20000 . Wu et al. calculated the number of infected people in Wuhan by the number of cases in January 2020 and predicted that the number of infected cases on January 25 would exceed 6000 [20]. In terms of epidemic prevention and control measures, Lin et al. proposed a conceptual model of the outbreak of COVID-19 in Wuhan, which was based on the consideration of such interventions as holiday extension, travel restriction, hospitalization, and quarantine [21]. Adam et al. estimated that within two weeks of the restriction, the transmission volume would decrease by nearly a half. The Lancet published an editorial that China has successfully avoided a large number of cases of infection and death because of the strong public countermeasures [22]. They also called on the other countries to learn from China's experience [23]. To sum up, the current research of COVID-19 primarily focuses on the prediction and estimation of epidemic development. There is a lack of modeling and analysis of specific prevention measures. Therefore, this paper focuses on analyzing the effectiveness of the measures such as closing communities, enforcing individual protection, isolating suspected cases, and establishing temporary treatment centers for mildly infected cases.

The dynamic models are utilized to study the effect of various prevention and control measures in the COVID-19 pandemic (He et al., 2020) [24]. For example, Zhao and Chen developed a SUQC model to explicitly parameterize the intervention effects of control measures of the pandemic [25]. Moran's spatial statistic with various definitions of neighbors was used by Kang et al. (2020) to conduct a test to determine whether a spatial association of the COVID-19 infections existed [26]. Linka et al. (2020) combined a global network mobility model with a local epidemiology model to simulate and predict the outbreak dynamics and control of the pandemic in Europe [27]. On this basis, Jia et al. (2020) developed a spatiotemporal risk source model, which can not only forecast the distribution of confirmed cases but also identify regions that have a high risk of transmission at the early stage of the pandemic [28].

This research combines the infectious disease model (hereinafter referred to as "SEIR") with the system dynamics model (hereinafter referred to as "SD"). We analyze the countermeasures of Wuhan by the combined model, and finally, propose feasible suggestions for the response to the epidemic. The model simulates the situation of a large area and scale of individuals within an acceptable complexity. Moreover, the Chinese official data are utilized to fit the model, while the values of the parameters in this model are set by the authoritative data. On this basis, the effectiveness of the model is verified. The remainder of this paper is organized as follows: The second section proposes the COVID-19 epidemic and its response system. The third 
section establishes the improved SEIR-SD model for the system. The fourth section makes simulations on several countermeasures according to the real and some hypothetical scenarios in Wuhan. The fifth section is the conclusions and suggestions.

\section{COVID-19 Epidemic and Its Response System}

The factors in the COVID-19 epidemic and its response forms a complex dynamic feedback system that includes the medical supplies, the hospital beds, the medical staff, the patients, etc. The system consists of the following subsystems: the infection subsystem, the quarantine subsystem, and the medical subsystem.

2.1. Infection Subsystem. In the infection subsystem, the total number of people in the city, the average number of contacts per person daily, and the infection rate have a positive impact on the number of people who are infected and enter the incubation period. The close contacts of patients in the incubation period are infected due to the lack of protective measures, resulting in an increase in the number of infected people daily. After the incubation period, patients begin to develop symptoms. In addition, the number of infected people, the average number of close contacts, and susceptible people were positively correlated with the number of close contacts of symptomatic people. Among the close contacts, some are infected and go into the incubation period, while others are uninfected but still susceptible. There are two important factors to determine whether close contacts are infected or not. The first one is the infection rate of close contacts. The transmission mode of the virus is diverse. It is easy to be infected without any protective measures. After taking measures such as wearing masks and disinfecting, the infection rate of close contacts is greatly reduced. The second one is the quarantine rate. If the infected cases are found precisely and quarantined in time, the probability of secondary infection should decrease significantly.

The causal relationship of the infection subsystem is shown in Figure 1. Table 1.

The meaning of parameters in Figure 1 is shown in

2.2. Quarantine Subsystem. In the quarantine subsystem, the factors that have a positive impact on the number of quarantined close contacts one day are the number of close contacts and the overall proportion of quarantined close contacts (the quarantined rate). Among these quarantined close contacts, some are diagnosed as infected and receive treatment, while others are not infected and released from quarantine. In addition to the number of quarantined close contacts on that day, the influencing factors of the number of these two parts also include the overall proportion of close contacts who are infected. The number of newly quarantined people on that day and the quarantine days positively impact the cumulative number of quarantined people and the number of released people on that day, while the number of

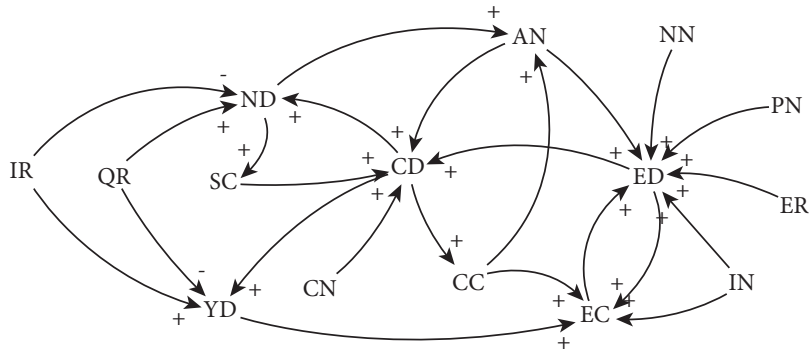

FIgURE 1: Causal relationship of infection subsystem.

released people on that day negatively impacts the cumulative number of quarantined people.

The causal relationship of the quarantine subsystem is shown in Figure 2.

The meaning of parameters in Figure 2 is shown in Table 2.

2.3. Medical Subsystem. In the medical subsystem, the patients who are infected in the incubation period have symptoms after the incubation period, so the number of patients who have symptoms after the incubation period is affected by the average incubation period of the disease and the number of patients who are infected and in the incubation period. Due to the influence of medical supplies and hospital beds, only a part of the people with symptoms can be admitted to the hospital. Therefore, the positive influencing factors of the number of people with symptoms admitted to the hospital one day are the number of people with symptoms and the overall proportion of people with symptoms admitted to the hospital. The number of hospitalized patients directly affects the number of cured patients and the number of deaths. Some of the people who have not been treated can recover by themselves. The number of selfhealing people is affected by the average self-healing time, the proportion of patients treated, and the number of patients with symptoms.

The causal relationship of the medical subsystem is shown in Figure 3.

The meaning of parameters in Figure 3 is shown in Table 3.

\section{Improved SEIR-SD Model for COVID-19 Epidemic and Its Response}

The classical SEIR epidemic model considers four groups of people including the susceptible, exposed, infected, and rehabilitated. It uses a set of differential equations to express the transmission mechanism of infectious diseases. But the model does not consider the strong intervention of management measures. The Chinese government and the local government of Wuhan have implemented a series of effective measures to control the spread of the virus. These measures include but are not limited to closing communities, enforcing individual protection, isolating suspected cases, and establishing temporary treatment centers for mildly infected cases. The spread of the virus has been largely 
TABle 1: Definition of parameters in Figure 1.

\begin{tabular}{lc}
\hline Parameter & Definition \\
IR & The proportion of close contacts infected \\
QR & The proportion of close contacts found and quarantined (quarantine rate) \\
ND & The number of uninfected cases in close contact but not quarantined daily \\
YD & The number of infected cases in close contact but not quarantined daily \\
CN & The average number of close contacts of infected cases \\
CD & The number of close contacts of those with symptoms daily \\
AN & The number of people except for those in quarantine, hospitalization, and death \\
CC & The cumulative number of close contacts \\
EC & The cumulative number of infected cases in the incubation period \\
ED & The number of infected cases going into incubation period daily \\
NN & The average number of daily contacts per person (average contact number) \\
PN & Cumulative population \\
ER & The proportion of infections after contacting infected cases (contact infection rate) \\
SC & Susceptible population \\
IN & The number of initial infected cases
\end{tabular}

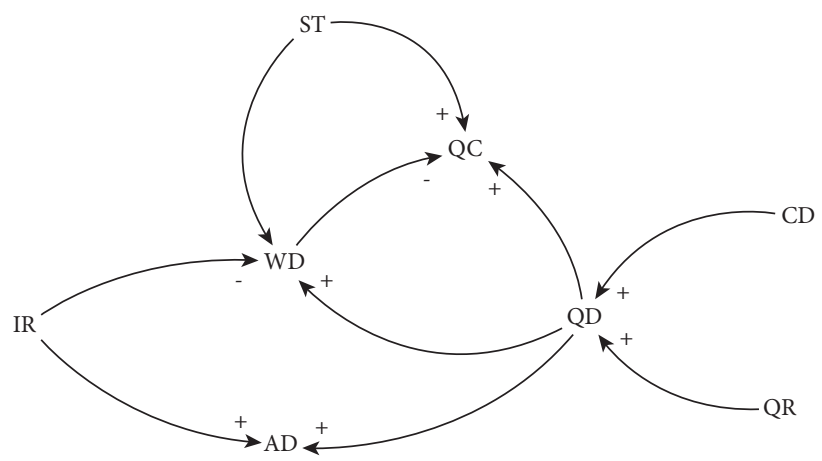

FIgURE 2: Causal relationship of quarantine subsystem.

TABle 2: Definition of parameters in Figure 2.

\begin{tabular}{lc}
\hline Parameter & Definition \\
\hline WD & The number of close contacts released from quarantine daily \\
AD & The number of infected cases in quarantine daily \\
QC & The cumulative number of infected cases in close contacts \\
QD & The number of quarantined close contacts daily \\
ST & Quarantine days \\
\hline
\end{tabular}

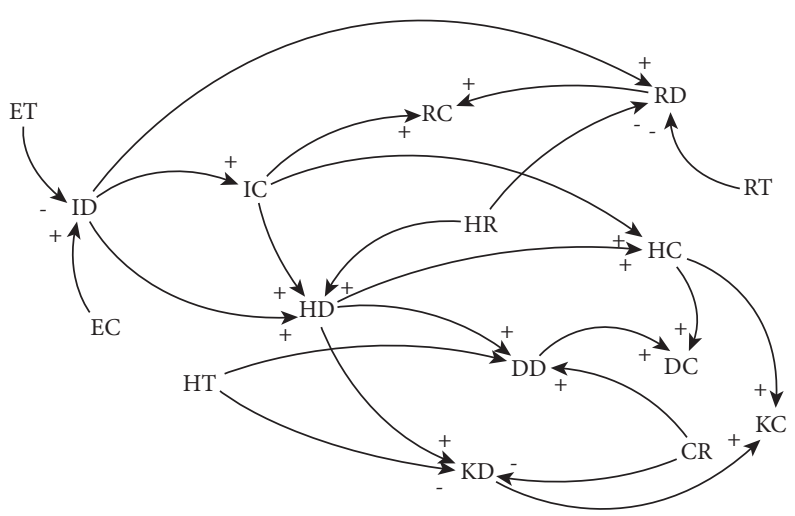

FIgURE 3: Causal relationship of the medical subsystem. 
TABLe 3: Definition of parameters in Figure 3.

\begin{tabular}{lc}
\hline Parameter & Definition \\
\hline ET & The average incubation period \\
ID & The number of new infections daily \\
IC & The cumulative number of infections \\
HD & The number of new infections and admitted to the hospital daily \\
HT & Average hospitalization time \\
RC & The cumulative number of self-healing \\
HR & The proportion of patients admitted to hospital (hospital admission rate) \\
DD & The number of cases cured and discharged daily \\
KD & The number of new deaths daily \\
RD & The number of self-healing daily \\
RT & Average self-healing time \\
HC & The cumulative number of cases in hospital \\
DC & The cumulative number of cases cured and discharged \\
CR & Average cure rate \\
KC & The cumulative number of deaths
\end{tabular}

controlled because of the interventions. This study intends to improve the classical SEIR model by adding the variables related to the management measures. Afterward, we combine the improved SEIR model with SD simulation to analyze the influence of management factors, such as the community closure degree, the personal protection degree, the hospital treatment rate, and the quarantine rate of close contacts on the number of confirmed cases and deaths of a country or region.

Based on the COVID-19 epidemic and its response system proposed in Section 2. We propose the flowchart of the infection subsystem (Figure 4), quarantine subsystem
(Figure 5), and medical subsystem (Figure 6). There are three types of variables in the flowcharts which are the level variables, rate variables, and auxiliary variables. The level variables in the rectangle represent the cumulative effect of the system over time. The rate variables in the form of a valve control the strength of the system change. Auxiliary variables are used to construct information feedback between the variables.

In the infection subsystem, CC, EC, and SC are selected as the level variables, while the rate variables are $\mathrm{CD}, \mathrm{ED}$, and ND. The functional relationships of the infection subsystem are shown in the following equations:

$$
\begin{aligned}
& \frac{\mathrm{d} C C}{\mathrm{~d} t}=C D-Y D-N D-Q D, \\
& \frac{\mathrm{d} E C}{\mathrm{~d} t}=E D+Y D-I D, \\
& \frac{\mathrm{d} S C}{\mathrm{~d} t}=N D+W D-E D-C D, \\
& A N=P N-K C-H C-Q C, \\
& E D= \begin{cases}S C, & \frac{(I C+E C) \cdot N N \cdot E R \cdot S C}{(I C+E C) \cdot N N \cdot E R \cdot S C} \\
\frac{A N}{A N}, & \frac{(I C+E C) \cdot N N \cdot E R \cdot S C}{A N} \leq S C,\end{cases} \\
& N D=C D \cdot(1-Q R) \cdot(1-I R), \\
& C D=\left\{\begin{array}{l}
S C-E D, \quad I D \cdot C N \geq S C-E D, \\
I D \cdot C N, \quad I D \cdot C N<S C-E D,
\end{array}\right. \\
& Y D=C D \cdot(1-Q R) \cdot I R,
\end{aligned}
$$




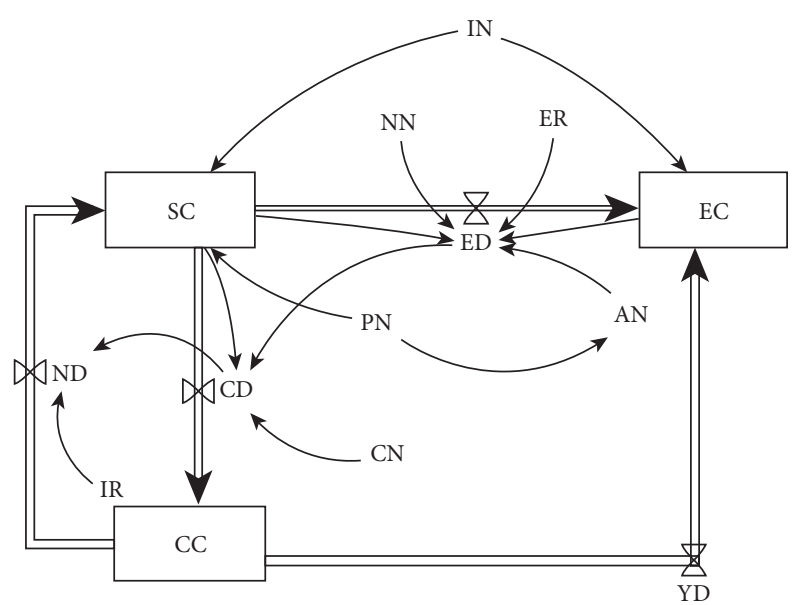

FIGURE 4: Infection subsystem flowchart.

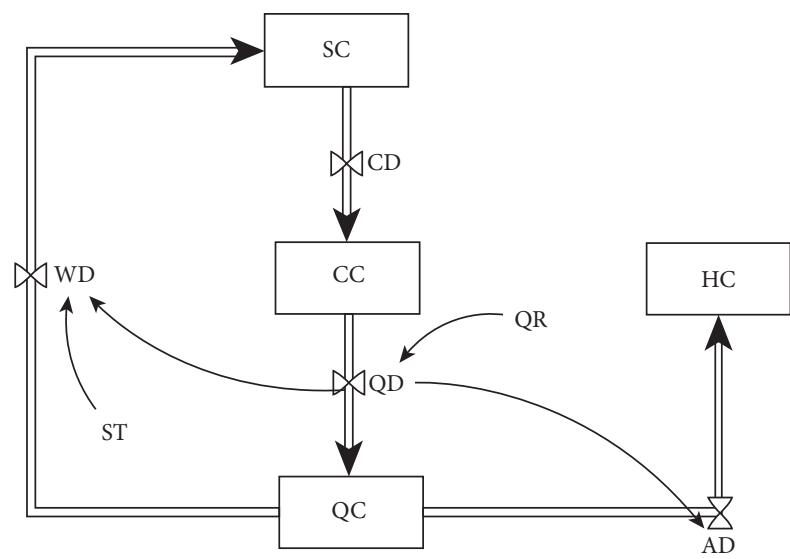

Figure 5: Quarantine subsystem flowchart.

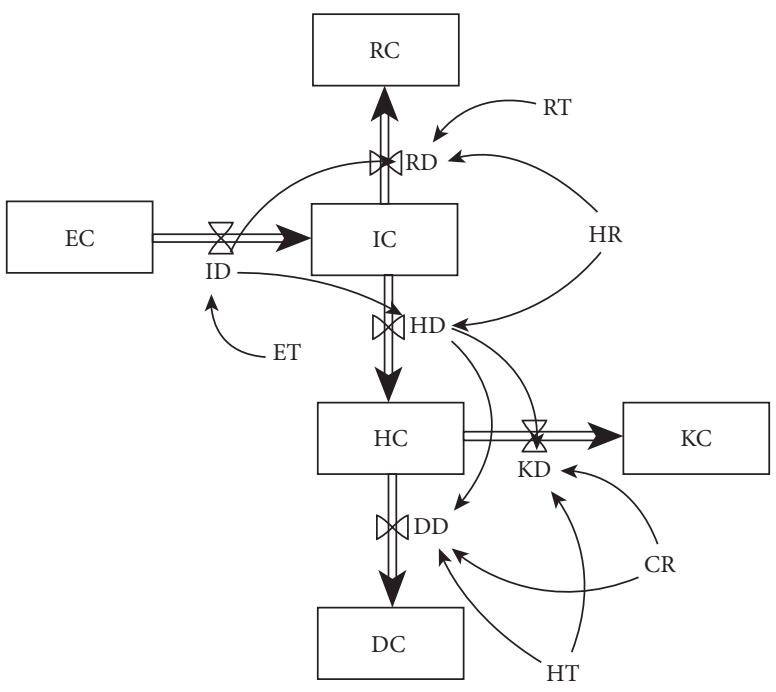

FIgURE 6: Medical subsystem flowchart.

In the quarantine subsystem, the level variable is QC, while the rate variables are $\mathrm{WD}, \mathrm{QD}$, and $\mathrm{AD}$. Equations (9)-(12) show the functional relationships of the quarantine subsystem.

$$
\begin{aligned}
& \frac{\mathrm{d} Q C}{\mathrm{~d} t}=Q D-W D-A D, \\
& A D= \begin{cases}Q D \cdot I R, & \text { time } \geq 1, \\
0, & \text { time }<1,\end{cases} \\
& Q D= \begin{cases}C D \cdot Q R, & \text { time } \geq 1, \\
0, & \text { time }<1,\end{cases} \\
& W D= \begin{cases}Q D \cdot(1-I R), & \text { time } \geq S T, \\
0, & \text { time }<S T,\end{cases}
\end{aligned}
$$

In the medical subsystem, the level variables are DC, IC, $\mathrm{HC}, \mathrm{KC}$, and $\mathrm{RC}$ which are determined by the rate variables $\mathrm{DD}, \mathrm{ID}, \mathrm{HD}, \mathrm{KD}$, and $\mathrm{RD}$. The functional relationships of the medical subsystem are shown in the following equations:

$$
\begin{aligned}
\frac{\mathrm{d} D C}{\mathrm{~d} t} & =D D, \\
\frac{\mathrm{d} I C}{\mathrm{~d} t} & =I D-R D-H D, \\
\frac{\mathrm{d} H C}{\mathrm{~d} t} & =H D+A D-D D-K D, \\
\frac{\mathrm{d} K C}{\mathrm{~d} t} & =K D, \\
\frac{\mathrm{d} R C}{\mathrm{~d} t} & =R D,
\end{aligned}
$$

$D D= \begin{cases}H D \cdot C R, & \text { time } \geq H T, \\ 0, & \text { time }<H T,\end{cases}$

$H D=I D \cdot H R$,

$$
\begin{aligned}
& I D= \begin{cases}E D, & \text { time } \geq E T, \\
0, & \text { time }<E T,\end{cases} \\
& K D= \begin{cases}H D \cdot(1-C R), & \text { time } \geq H T, \\
0, & \text { time }<H T,\end{cases} \\
& R D= \begin{cases}I D \cdot(1-H R), & \text { time } \geq R T, \\
0, & \text { time }<R T,\end{cases}
\end{aligned}
$$

The improved SEIR-SD model is established, of which the system flowchart is shown in Figure 7 which consists of three parts, i.e., infection part, quarantine part, and medical part. The model considers asymptomatic infection and subclinical cases. The assumptions of the model include: there is only human to human transmission; there is no specific medicine or vaccine for the virus; other diseases do not influence the mortality rate and other parameters; there is no population mobility; deaths occur only in hospital; the isolated cases are immediately tested for nucleic acid 


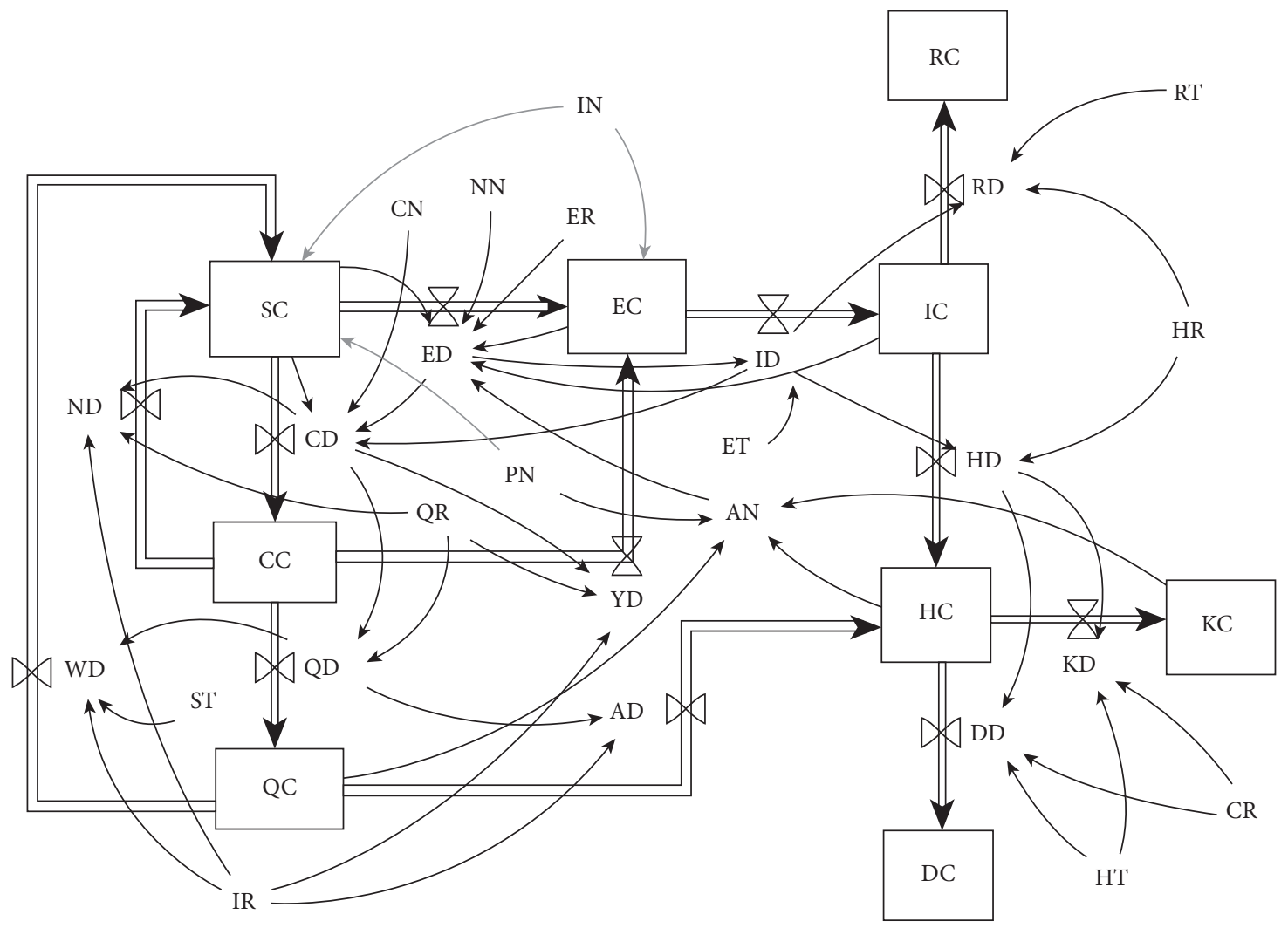

FIGURE 7: System flowchart of the improved SEIR-SD model.

regardless of the existence of symptoms; the close contacts are quarantined within one day if the conditions permit. The isolated cases are tested for nucleic acid within one day. In this model, the initial values of the level variables are zero except SC and EC. The sum of the initial values of SC and EC is equal to the cumulative population, and the initial value of $\mathrm{EC}$ is equal to IN.

\section{Simulation Results and Analysis}

\subsection{Simulation of Epidemic Responses in Wuhan}

4.1.1. Simulation Parameter Setting. This section tests the simulation effect of the model to ensure the validity and reliability of the model in terms of structure, parameter settings, and functional relationships using the historical data of the COVID-19 epidemic in Wuhan. It was not until February 14th that the number of nucleic acid tests that can be performed in Wuhan every day met the demand. In other words, the number of confirmed cases was reliable after that. Therefore, the initial time of the model is set as February 14th, 2020. The basic parameters of the model are set as follows: initial time $=1$, end time $=100$, time step $=1$.

The parameter values of the model are set based on the official data or authoritative research results that have been published, as shown in Table 4 [22, 29-33].
We set $\mathrm{QR}=0.94, \mathrm{HR}=0.9$ based on the data published by Hubei Provincial Health Committee and Wuhan $\mathrm{Mu}-$ nicipal Health Committee.

According to the official announcement ([16, 34-37]), the following variable values are determined. In the initial period of the epidemic, residents can move freely in the city, so we set $\mathrm{NN}=2.7$ for this period. Since February 5th (time $=35$ ), villages, communities, and units have been closed, so we set $\mathrm{NN}=2$ for this period. After mid-February (time $=44)$, all the residential districts had been strictly closed, so we set $\mathrm{NN}=0.7$ for this period. According to the authoritative news report, in the early stage, there were insufficient personal protective supplies, such as masks, so we set $\mathrm{ER}=0.06$ for this scenario; in late February (time$=55$ ), the supplies gradually became sufficient, so we set $\mathrm{ER}=0.03$ for this scenario. We set $\mathrm{CR}=0.93$ based on the data published on the official website of Wuhan Health and Health Commission for the scenario in the early stage. In the later period (time $>38$ ), the public places are requisitioned to build temporary treatment centers and many designated hospitals are renovated to increase available beds, which effectively improves the treatment rate and cure rate, so we set $\mathrm{CR}=0.98$ for this scenario.

According to the policy and actual situation, the logical functions of NN, ER, and CR can be established as follows: 
TABLE 4: Model parameter values.

\begin{tabular}{lcccccccc}
\hline name & HT & CN & ET & RT & PN & Ir & In & ST \\
\hline Value & 12 & 2 & 5.2 & 9 & 10000000 & 0.17 & 1180 & 14 \\
\hline
\end{tabular}

$$
\begin{aligned}
N N & =\text { IF THEN ELSE }(\text { Time }<=35,2.7, \text { IF THEN ELSE }(\text { Time }>35: \text { AND: Time }<44,2,0.7)) \\
E R & =\text { IF THEN ELSE }(\text { Time }<=55,0.06,0.03), \\
C R & =\text { IF THEN ELSE }(\text { Time }<=38,0.93,0.98) .
\end{aligned}
$$

4.1.2. Simulation Results. The number of confirmed cases in Wuhan officially released by the authority cannot reflect the actual infection number due to the poor nucleic acid testing ability in the early stage of the epidemic. We select a total of 30 days from February 14th to March 14th for simulation. The simulated number of infections daily (ID) and a simulated cumulative number of deaths (KC) are compared with the actual data to verify the effectiveness of the model. The simulation results are shown in Figure 8.

As shown in Figure 8(a), the number of confirmed cases has been steadily decreasing since mid-February until a sharp decline occurred on February 19th. This is because all the cases were admitted and all the close contacts were quarantined compulsorily in Wuhan after February 16th. Figure 8(b) shows that as the cure rate increases, the cumulative number of deaths shows a slower trend.

From the simulation results, the average deviation and its variance between the actual and simulated number of confirmed cases in every single day are $-5.24 \%$ and 0.0516 , respectively. The average deviation and its variance between actual and simulated cumulative deaths are $-0.80 \%$ and 0.0013 , respectively. Therefore, it suggests that the improved SEIR-SD model proposed in this paper is effective.

During the epidemic, Wuhan has adopted the measures such as closing communities, enforcing individual protection, enhancing treatment rate (establishing temporary treatment centers for mildly infected cases, adding hospital beds, and adding medical personnel and supplies), and enhancing quarantine rate (accurate tracking and quick quarantine of close contacts and suspected cases). To study the effect of these measures on the stemming and control of this epidemic, this paper utilizes the improved SEIR-SD model to make simulations according to the measures.

\subsection{Simulation Analysis of the COVID-19 Epidemic Response Measures}

4.2.1. Impact of Hospital Administration Rate. HR is an important factor affecting the development of the epidemic. Based on the improved SEIR-SD model proposed in the previous section, this section studies the trend of ID and $\mathrm{KC}$ with time (T) under different HRs. In this simulation, it is assumed that residents can move freely $(\mathrm{NN}=3)$ while taking extremely limited personal protective measures $(E R=0.06)$, and other parameter values are the same as those proposed in Section 4.1. HR and $T$ are regarded as the experimental variables, while other variables are regarded as the control variables. The value of HR is set between 0 and 1 , while $T$ is between 0 and 30 (days). The simulation results are shown in Figure 9.

Figure 9(a) shows the trend of ID over time under different HRs. It can be seen that ID continues to grow with time, which gives rise to the effect of improving HRs increasingly more obvious. While HR is low, the epidemic spreads increasingly more rapidly, resulting in a substantial increase in the number of infected people; in the middle and late stage of the epidemic, ID has a maximum growth rate and exceeds 200000; after the majority of residents are infected, the growth rate declines. When HR is close to 1 , ID increases slowly, and the maximum value is only 16071 . Consequently, the growth rate of ID varies greatly under different HRs.

Figure 9(b) shows the trend of KC over time under different HRs. Overall, KC shows an exponential upward trend with time. When HR is lower than 0.5 , the number of patients that can be accommodated in the hospital is small, and most of the patients who died from infection are not included in the statistics. Therefore, $\mathrm{KC}$ is small in the figure. When HR exceeds $0.5, \mathrm{KC}$ shown in the figure is the actual value. It implies that with the increase of HR, the rising trend of KC gradually decreases.

Therefore, ensuring the adequate supply of medical materials and medical staff, which improves the hospital admission rate, can restrain the development of the epidemic to a great extent. Especially in the early stage of the epidemic, ensuring a high admission rate can effectively prevent largescale outbreaks.

4.2.2. Impact of Quarantine Rate. Close contacts of infected cases are important carriers of the virus. Therefore, their quarantine rate directly affects the development of the epidemic. This section studies the trend of ID and KC with time $(T)$ under different QRs. The basic assumptions of this simulation are as follows: residents can move freely $(\mathrm{NN}=3)$, the majority of infected cases are admitted to the hospitals $(\mathrm{HR}=90 \%)$, and few personal protection measures are taken $(\mathrm{ER}=0.06)$. $\mathrm{QR}$ and $T$ are regarded as the experimental variables, while other variables are regarded as the control variables. The value of QR is set between 0 and 1 , while $T$ is between 0 and 30 (days). The simulation results are shown in Figure 10.

Figure 10(a) shows the trend of ID over time under different QRs. With the passage of time, the number of infected cases increases gradually, and the effect of measures 


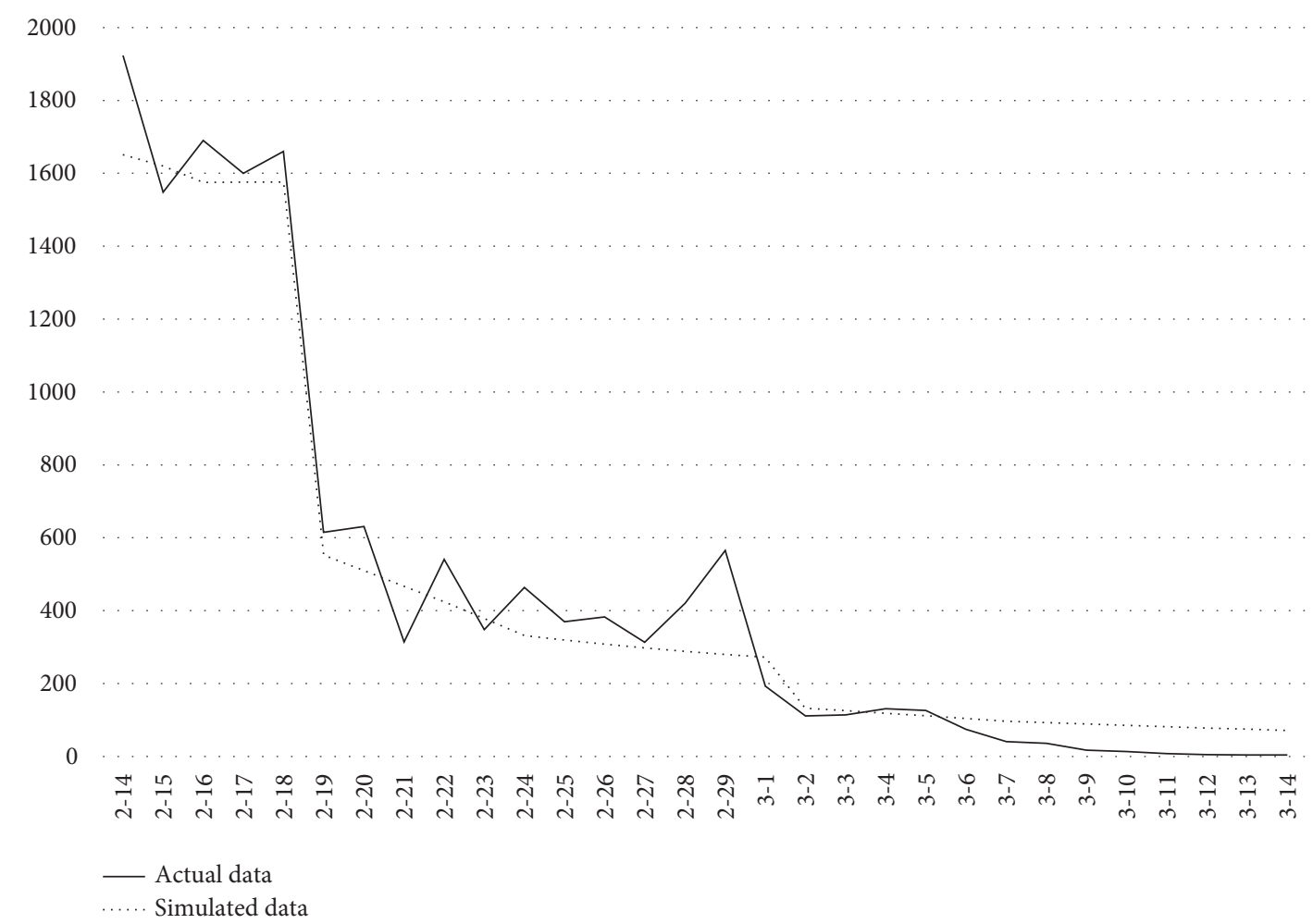

(a)

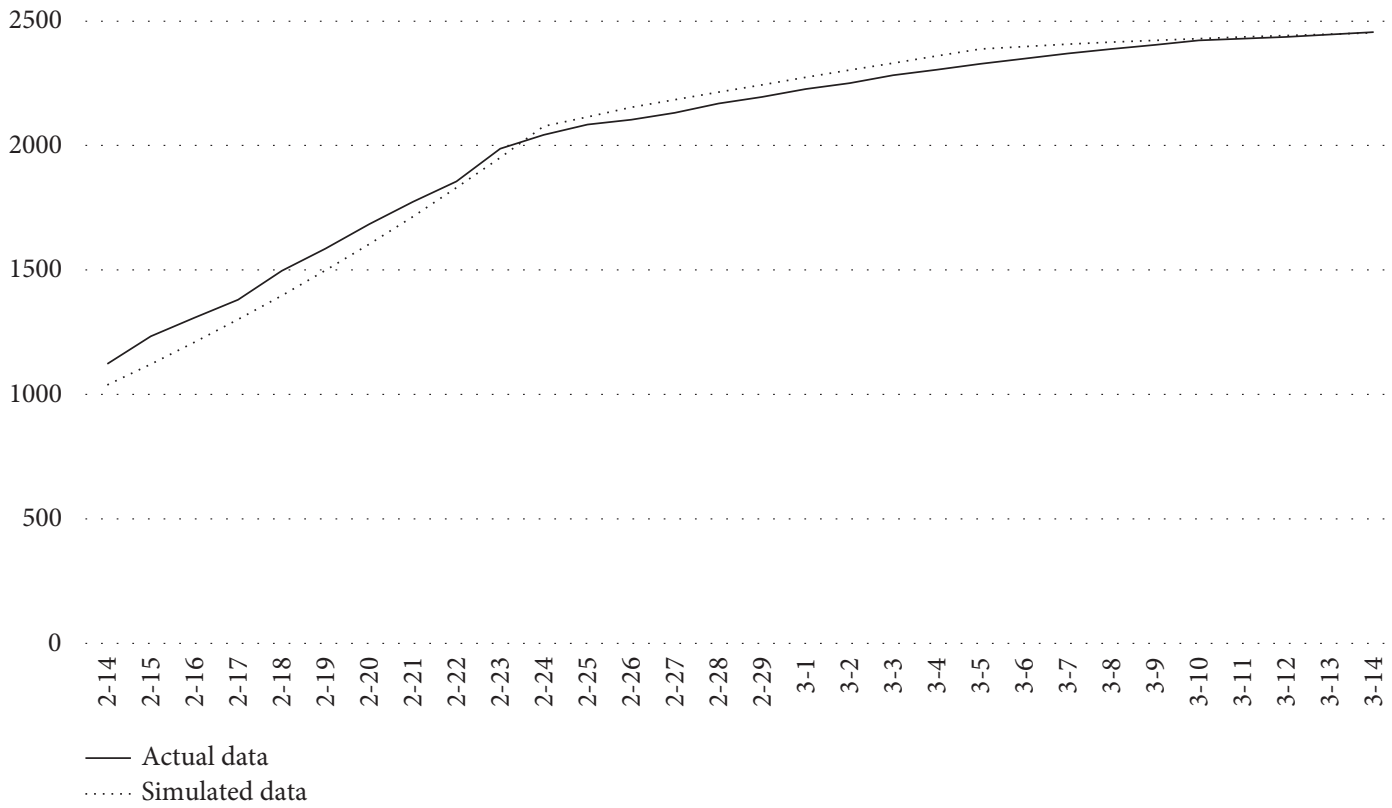

(b)

FIGURE 8: Comparison of simulated and actual data of the COVID-19 in Wuhan. (a) The number of confirmed cases in every single day. (b) The cumulative number of deaths.

to improve $\mathrm{QR}$ is more obvious. When $\mathrm{QR}$ is relatively low, the speed of disease transmission is the fastest. In this case, ID would reach 202368 on the 30th day. With the gradual increase of QR, the growth of confirmed cases slows down. When QR reaches 0.9, the upper limit of ID is only 19872.
Therefore, there are great differences in the growth of ID under different QRs.

Figure 10(b) shows the trend of $\mathrm{KC}$ over time under different QRs. As is shown in the figure, KC increases exponentially with time. When QR is low, the upper limit value 


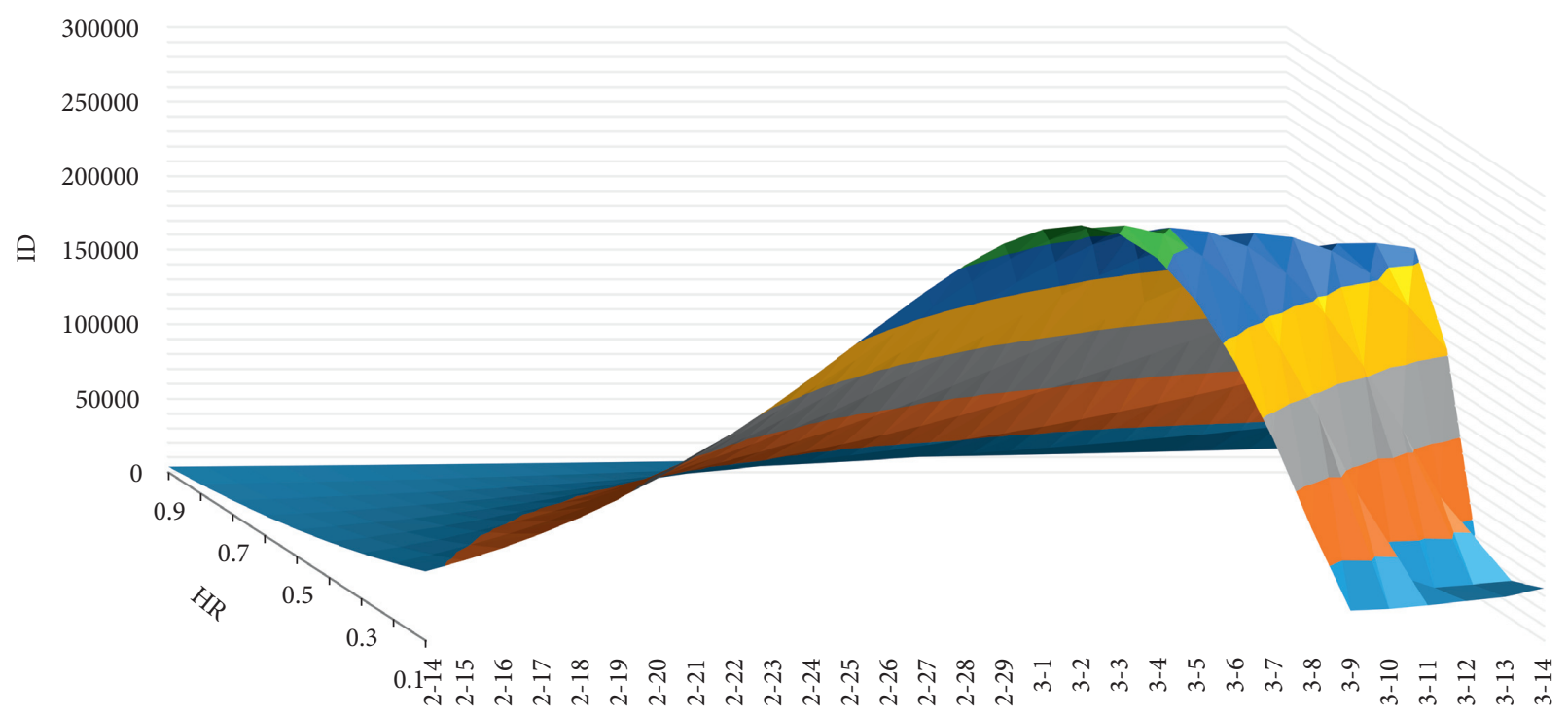

$\mathrm{T}$

(a)

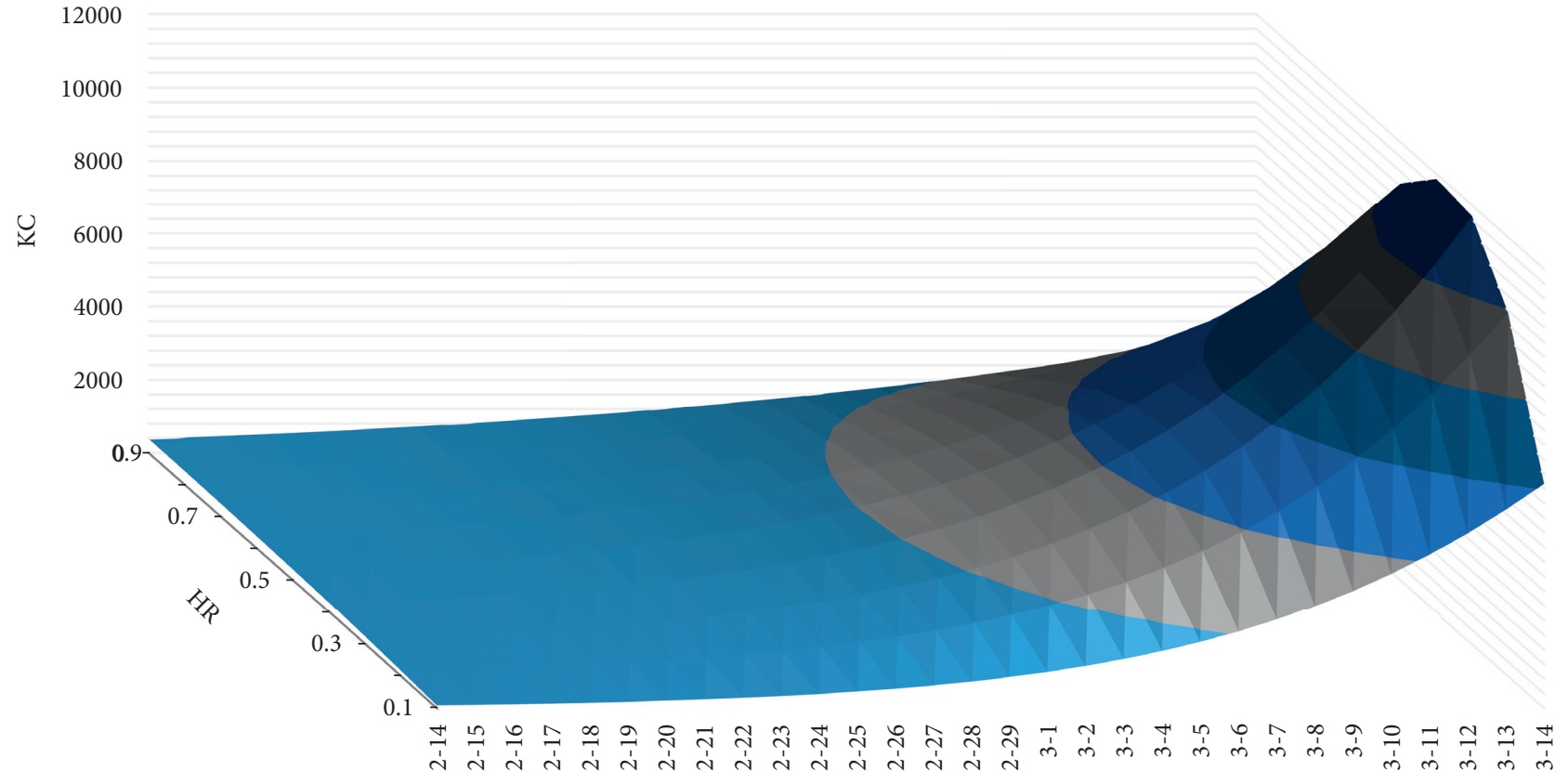

$\mathrm{T}$

(b)

FIgure 9: Trend of ID and KC under different HRs.

of $\mathrm{KC}$ is 13718 . By increasing QR, which inhibits the spread of the virus, the growth rate of $\mathrm{KC}$ decreases continuously. When QR reaches 0.9, KC should be lower than 3660. Consequently, with the increase of $\mathrm{QR}$, the rising trend of $\mathrm{KC}$ gradually decreases.

Therefore, improving the quarantine rate by accurate tracking and rapid quarantine of close contacts of infected cases can effectively reduce the number of infections and deaths. In the early stage of the epidemic, the improvement of the quarantine rate has a more significant impact on the spreading speed of the epidemic.
4.2.3. Impact of Average Contact Number. The degree of community closure directly determines the average contact number of residents. In this section, we study the trend of ID and $\mathrm{KC}$ with time $(T)$ under different NNs. The basic assumptions of this simulation are as follows: most infected cases are admitted to the hospitals $(\mathrm{HR}=90 \%)$, residents can move freely $(\mathrm{NN}=3)$, and few personal protection measures are taken $(\mathrm{ER}=0.06)$. NN and $T$ are regarded as the experimental variables, while other variables are regarded as the control variables. The value of NN is set between 0 and 3, while $T$ is between 0 and 30. The simulation results are shown in Figure 11. 


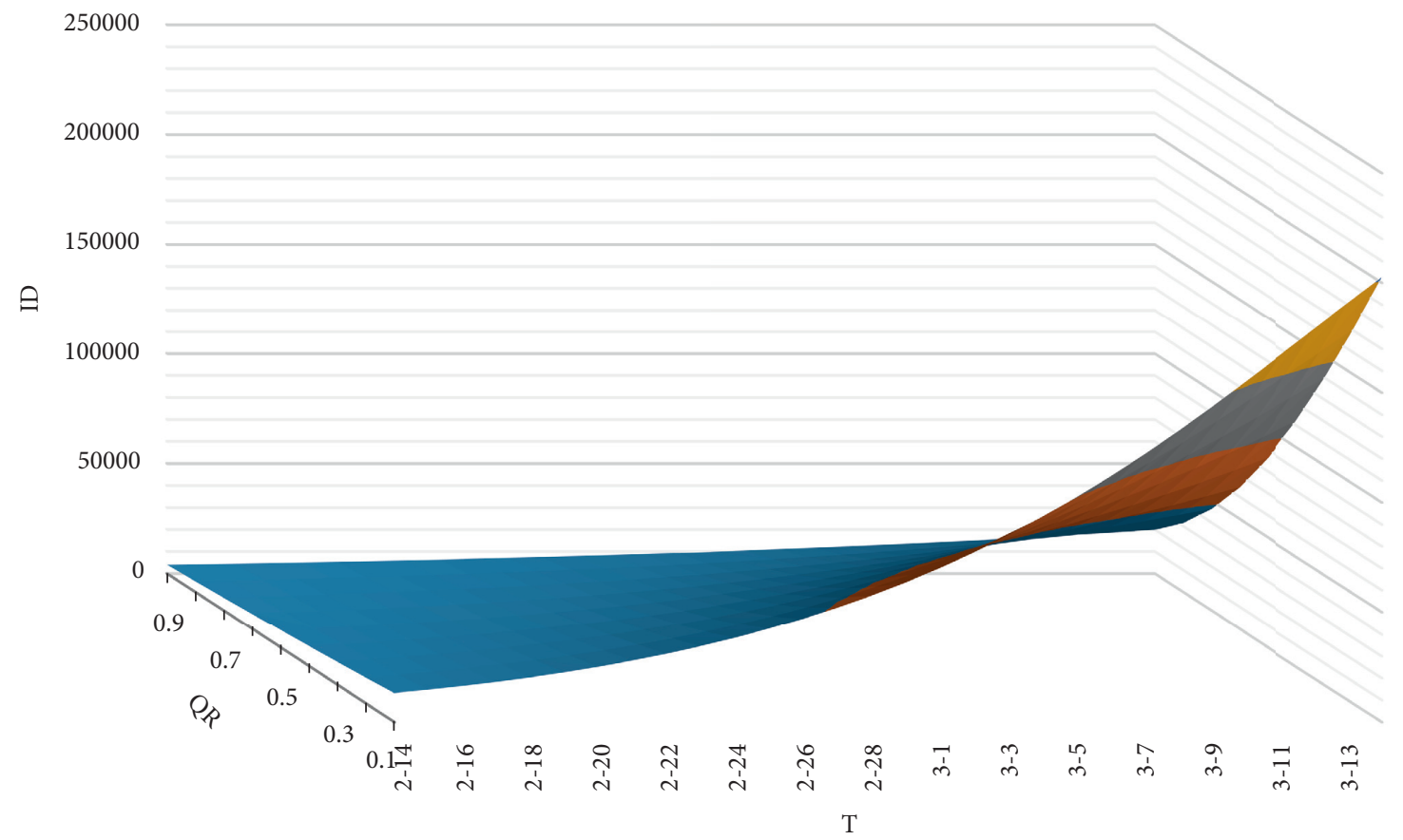

(a)

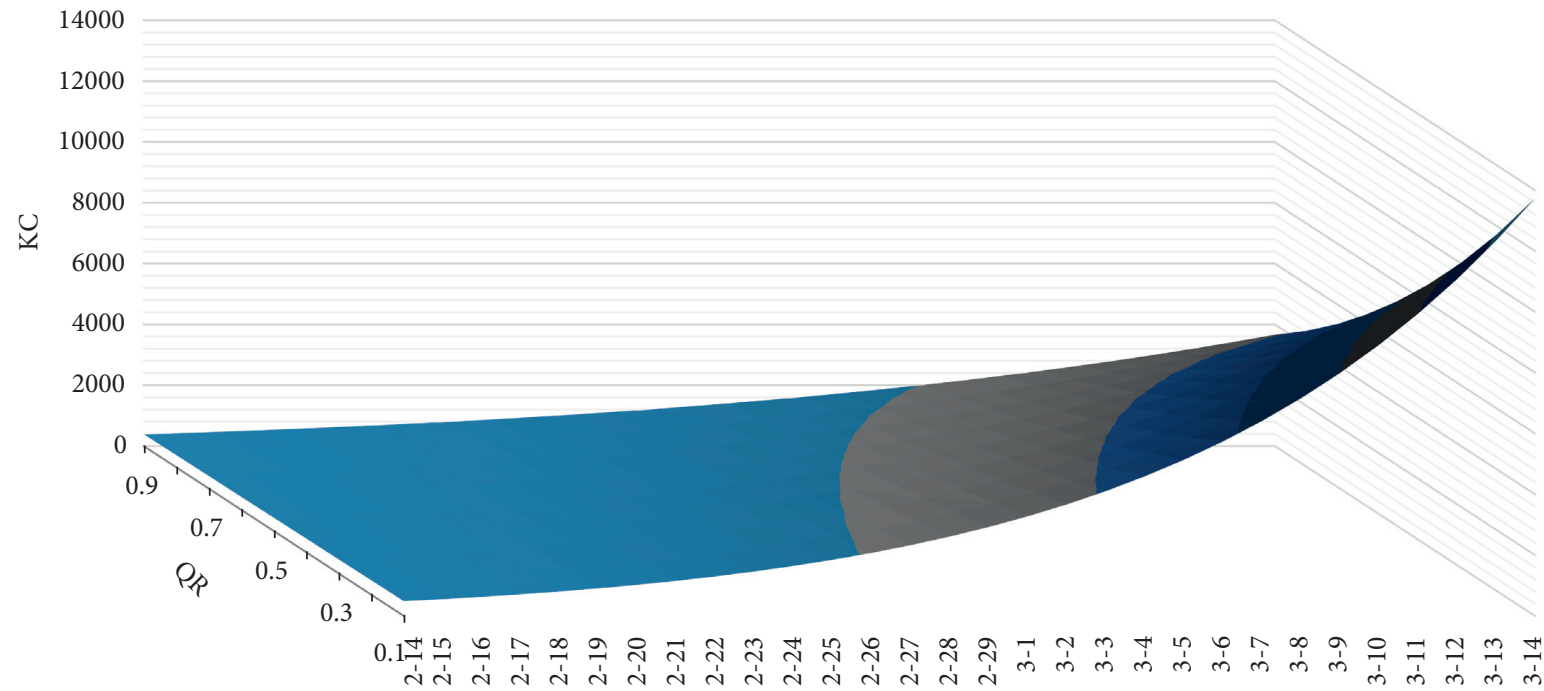

(b)

Figure 10: Trend of ID and KC under different QRs.

Figure 11(a) shows the trend of ID over time under different NNs. It is not difficult to find that the effect of early adoption of strict community closure measures is extremely obvious. In the case of the free flow of people, $\mathrm{NN}$ is the highest, so the epidemic spreads most rapidly. After 30 days, the value of ID reaches 16097 . In the case that the government takes compulsory community closure measures, ID decreases significantly, and the maximum number is only 24 , which differs significantly from the former case.

Figure 11(b) shows the trend of KC over time with different NNs. Under the condition of the free flow of residents, $\mathrm{KC}$ increases exponentially over time, reaching the maximum of 3358 cases. On the contrary, taking the greatest degree of community closure measures leads to a significant decline in the number of deaths (the maximum is only 31 cases). Consequently, strict closure measures can effectively reduce $\mathrm{KC}$.

It implies that the restraint effect of the epidemic should be very significant if strict community closure measures are taken in the early stage of the outbreak. Otherwise, the number of patients would increase exponentially, and the epidemic should be much more difficult to restrain. 


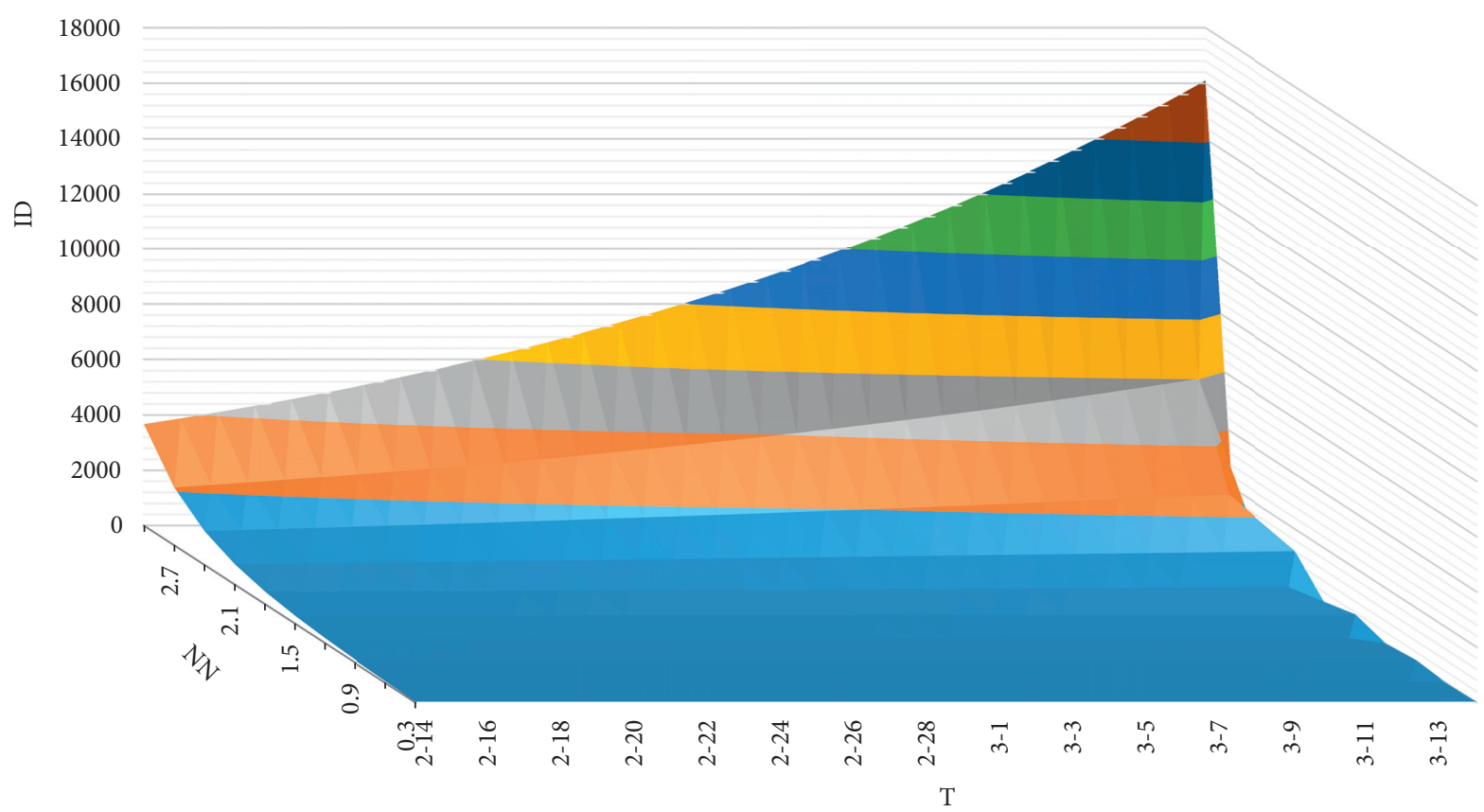

(a)

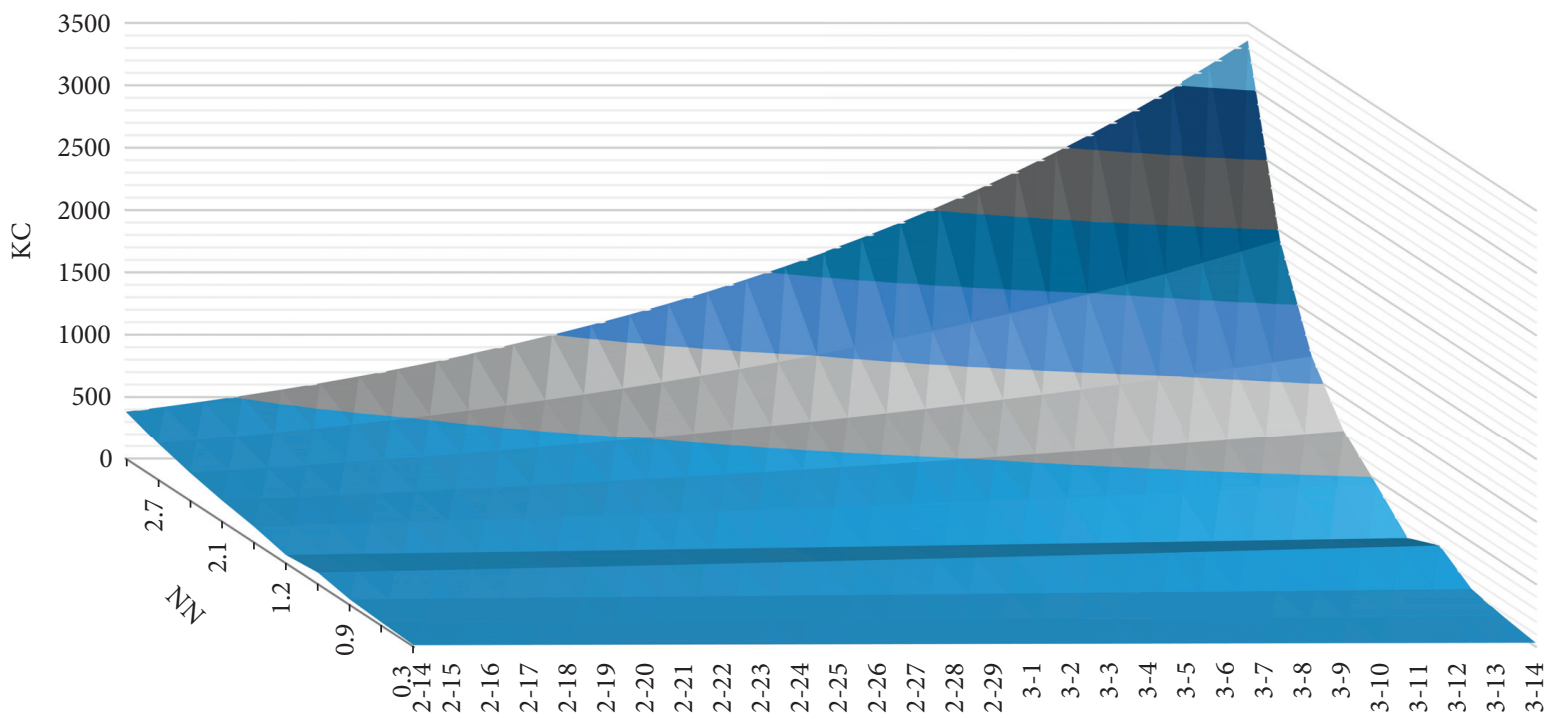

$\mathrm{T}$

(b)

Figure 11: Trend of ID and KC under different NNs.

4.2.4. Impact of Contact Infection Rate. In this section, we analyze the impact of ER on ID and KC. In the simulation, QR and HR are set to 0.94 and 0.9 , respectively. While people do not take any protective measures, and the contact infection rate is very high. In this case, the maximum value of ER is set to 0.06 . By contrast, in the case that people take the most stringent personal protection, ER reaches the minimum (0.03). Therefore, the value of ER is set between 0.030 and 0.060 . The simulation results are shown in Figure 12.

Figure 12(a) shows the trend of ID over time under different ERs. At the same level of ER, ID increases with the passage of time. While ER is 0.06 , the value of ID on the $30^{\text {th }}$ day of the epidemic can reach four times that at the beginning of the epidemic. At the same time node, the greater the ER, the more the ID. Additionally, with the increase of ER, the increase of ID becomes faster. While ER is 0.042 , ID reaches 1000 . While ER reaches 0.06 , ID could be more than 10000.

Figure 12(b) shows the trend of $\mathrm{KC}$ over time under different ERs. At the same level of ER, KC increases with time. While ER is $0.06, \mathrm{KC}$ could increase from 382 to 3359 in 30 days. At the same time node, the greater the ER, the more the $\mathrm{KD}$ and $\mathrm{KC}$. By contrast, while the ER is reduced to $0.045, \mathrm{KC}$ would also reduce to less than 1000 . 


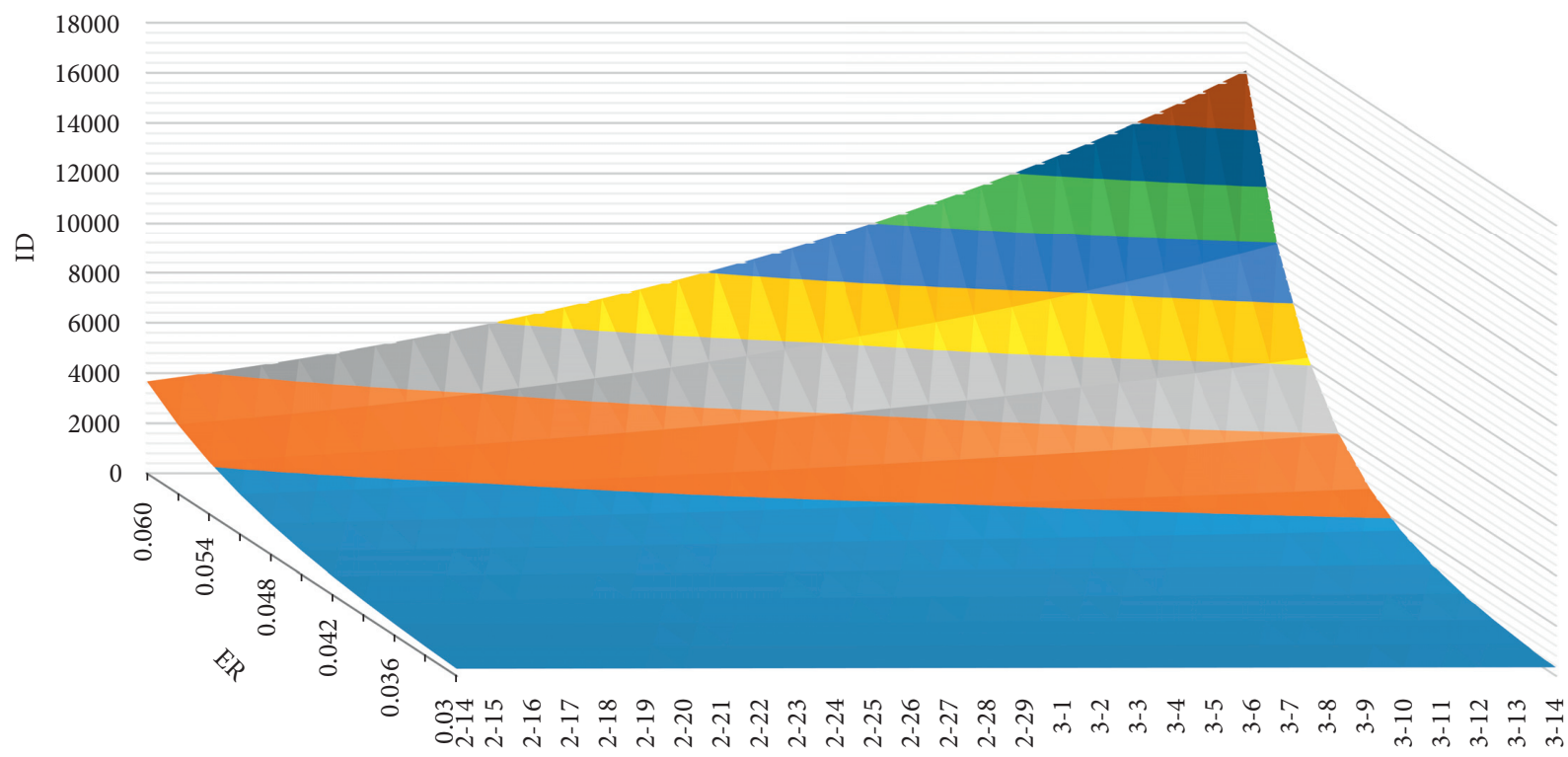

$\mathrm{T}$

(a)

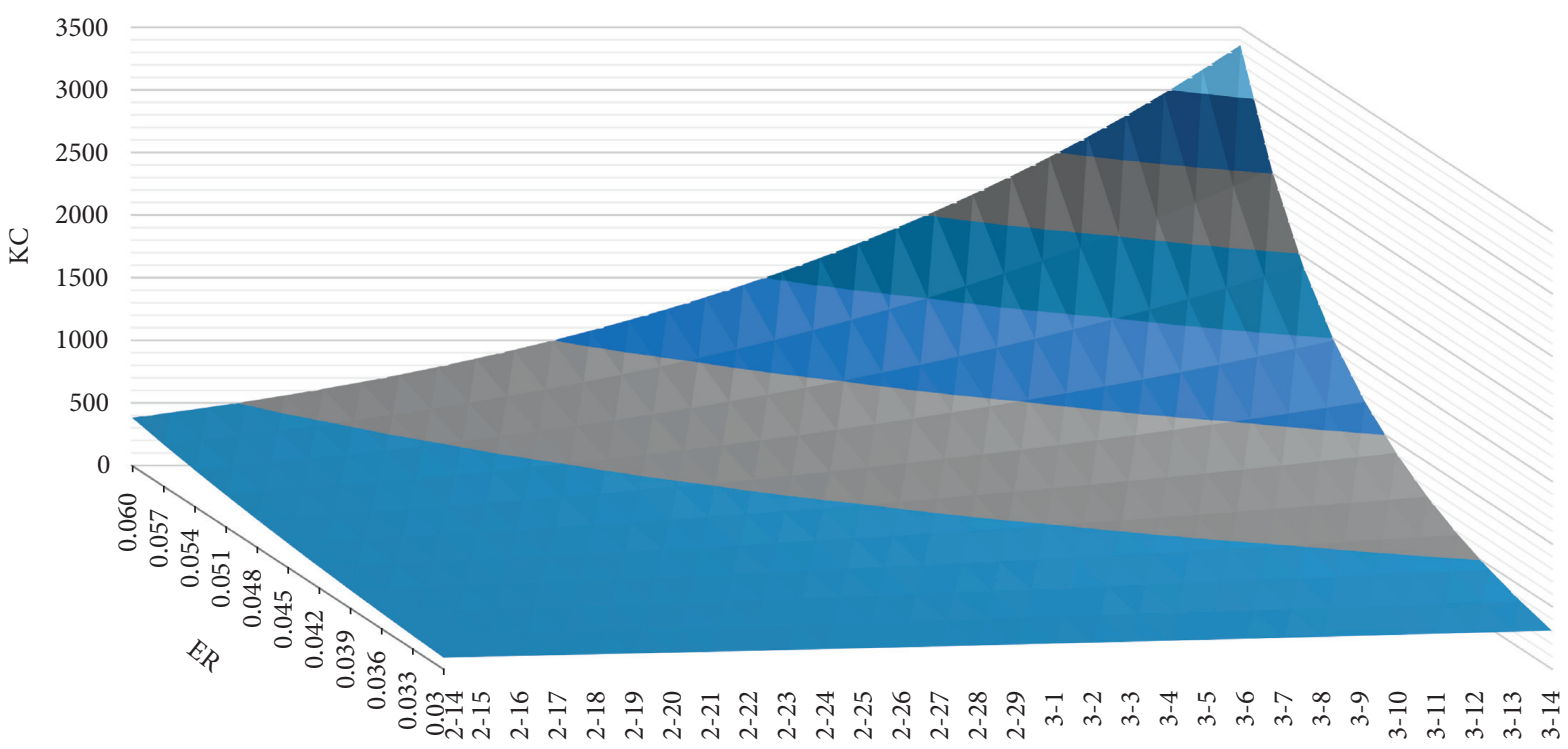

(b)

FIgURE 12: Trend of ID and KC under different ERs.

The simulation results show that when the contact infection rate is high, the number of new infections daily and the total number of deaths increase exponentially. Therefore, the measures such as wearing masks to reduce the contact infection rate are of pivotal importance to restrain the development of the epidemic.

4.2.5. Combined Impact of Hospital Admission Rate and Quarantine Rate. This section studies the values of IC and KC on the 30th day under different HRs and QRs. In this simulation, we assume that residents can move freely
$(\mathrm{NN}=3)$ and take extremely limited personal protection measures $(E R=0.06)$. $H R$ and $Q R$ are regarded as the experimental variables, while other variables are regarded as the control variables. The value of QR is set between 0 and 1 , while $T$ is between 0 and 30 . The simulation results are shown in Figure 13.

Figure 13(a) shows the values of IC under different HRs and QRs. Given a fixed value of QR, with the increase of $H R$, IC continues to decline. Specifically, if both $Q R$ and HR are 0.1 , then IC is 6636770; while HR is increased to 0.9 , IC decreases to 1151102. Additionally, Given a fixed value of $\mathrm{HR}$, with the increase of QR, IC decreases. Specifically, while 


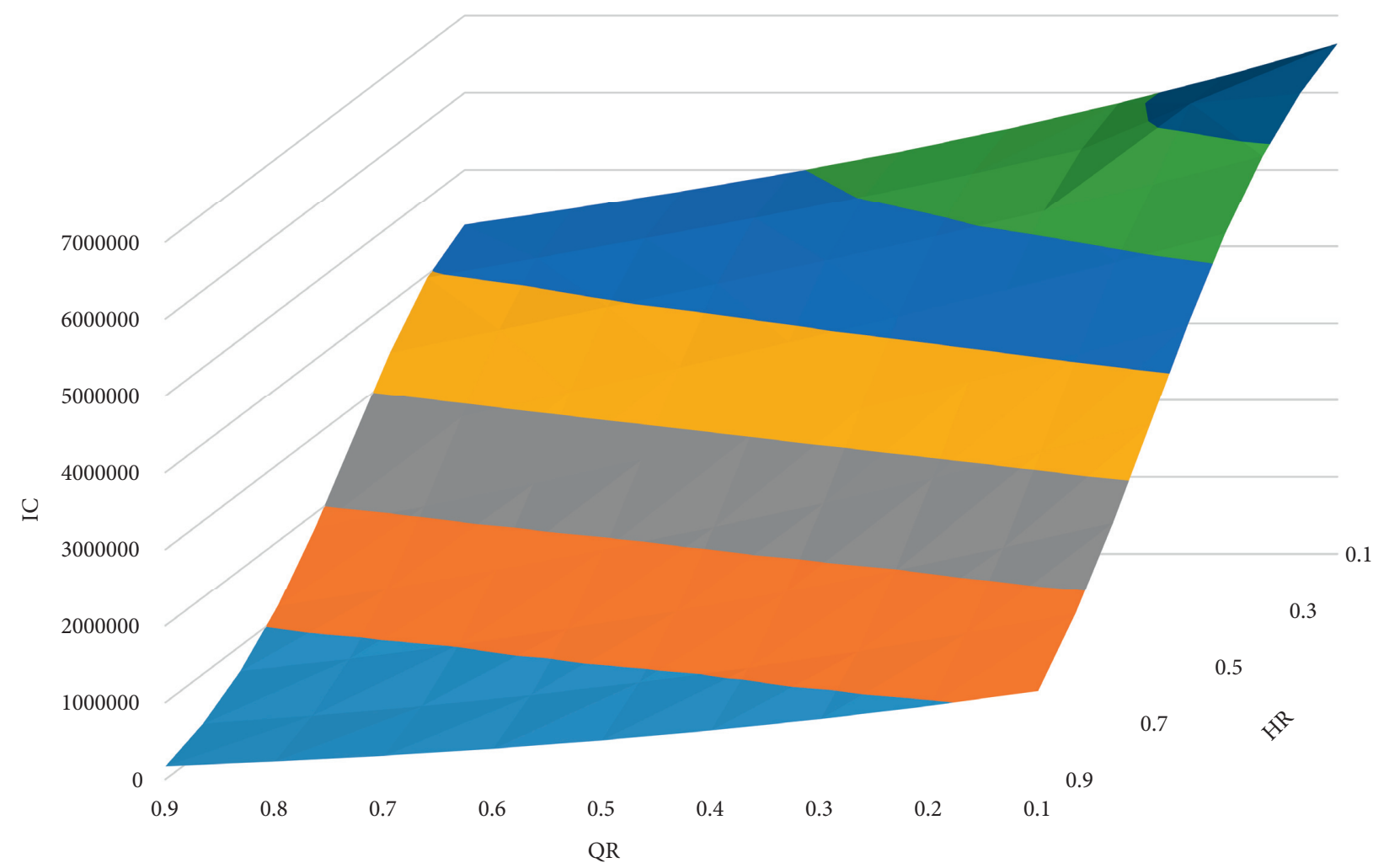

(a)

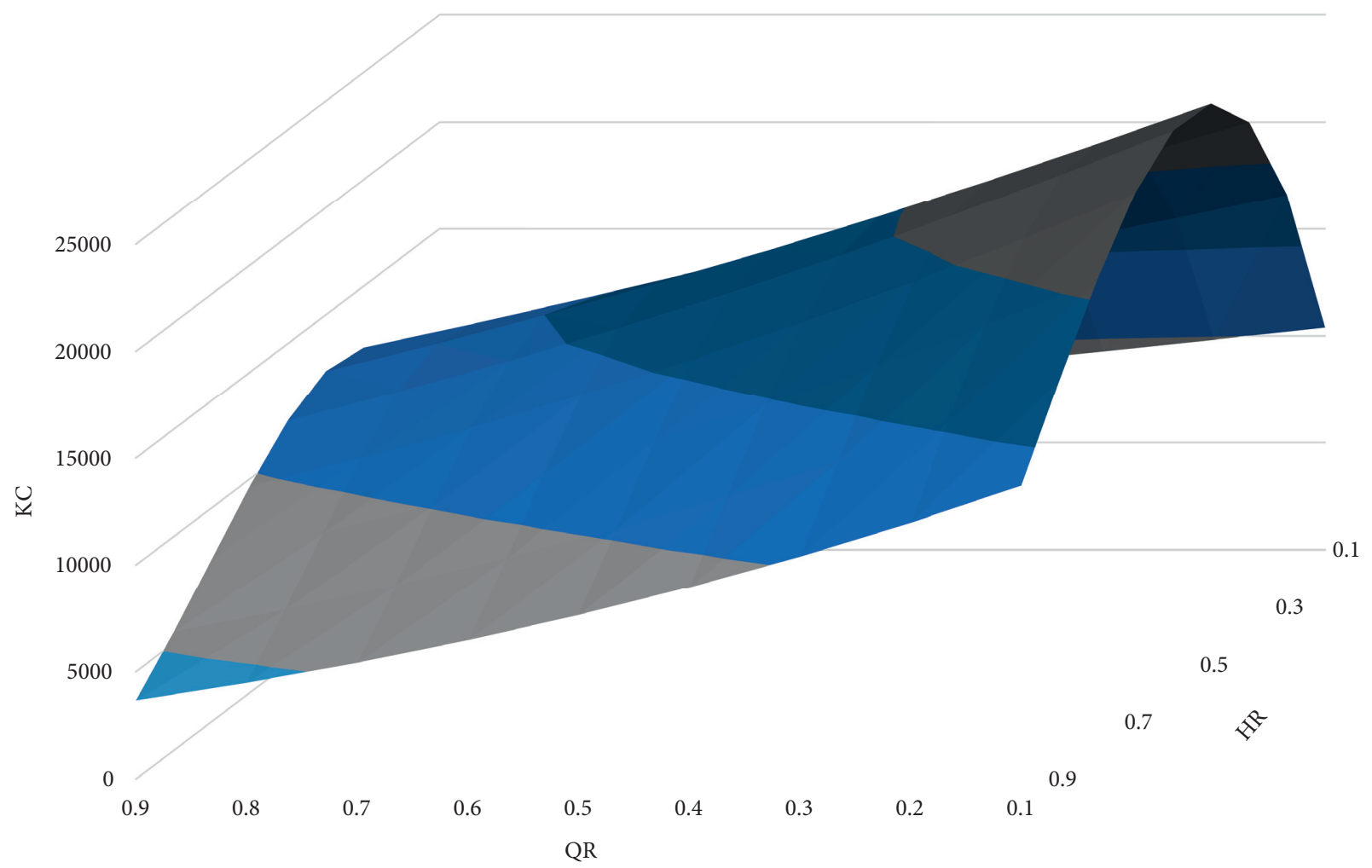

(b)

FIgure 13: Values of IC and KC under different HRs and QRs. 


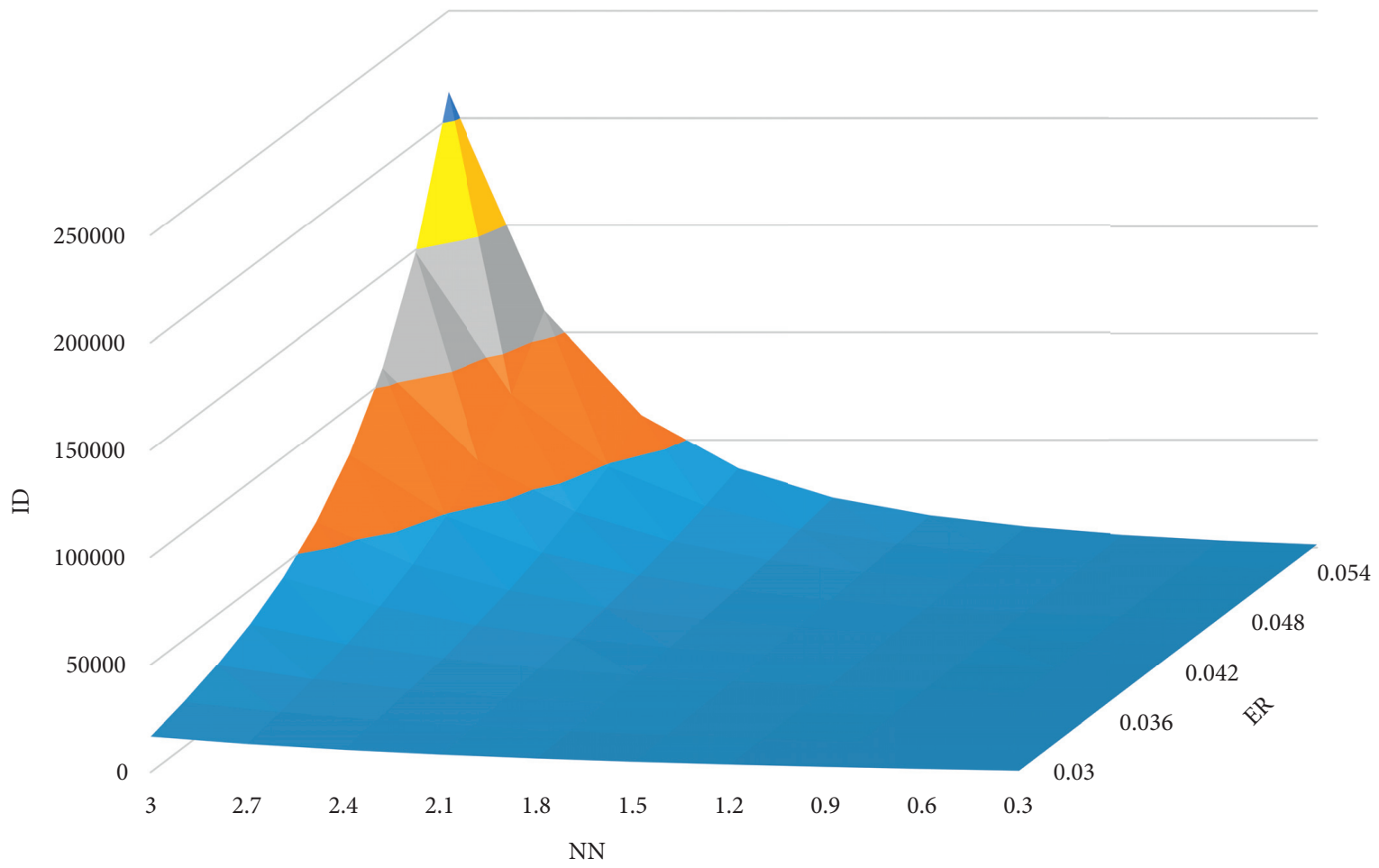

(a)

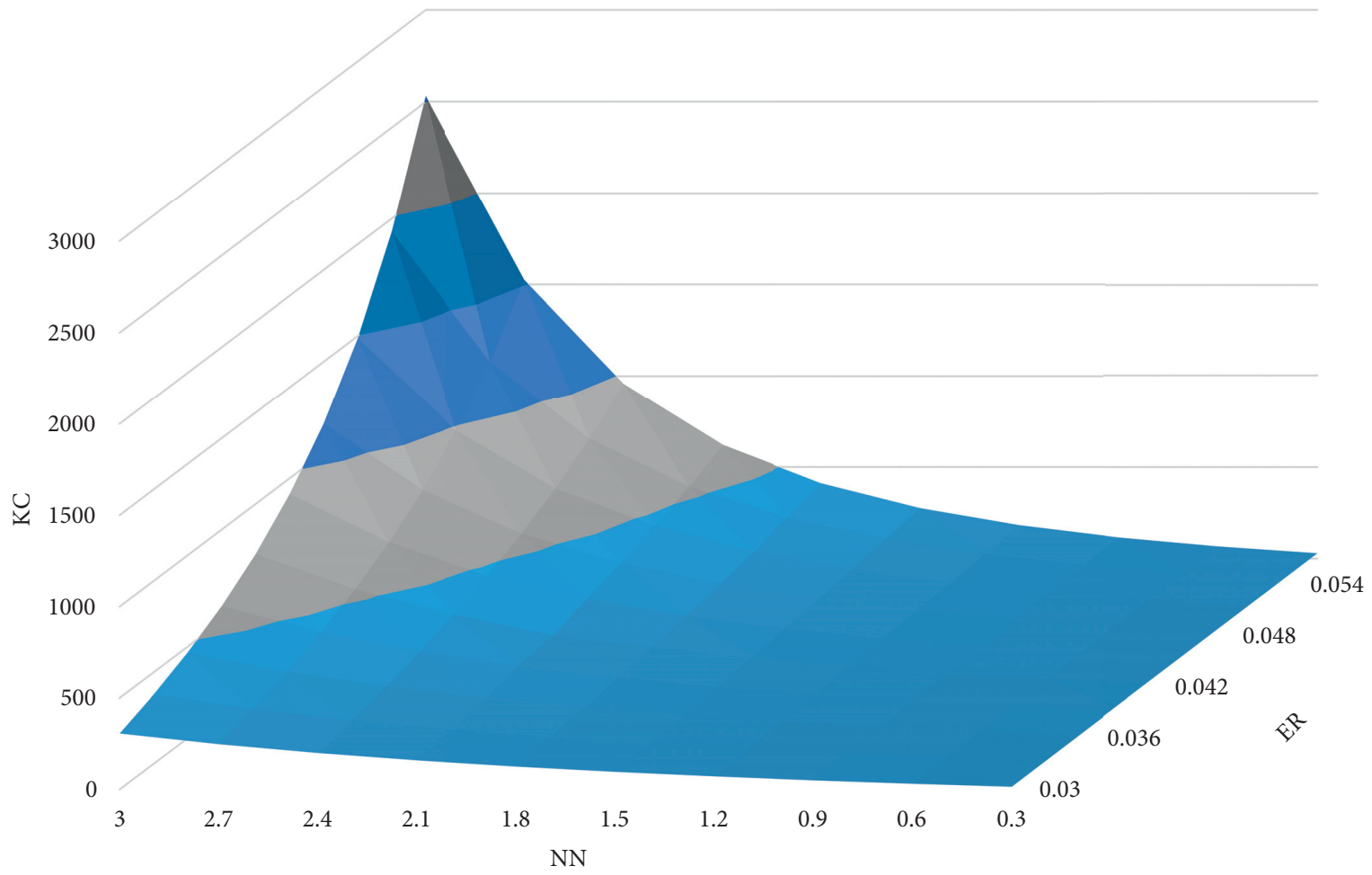

(b)

Figure 14: Values of IC and KC under different NNs and ERs.

HR is 0.1 and QR is 0.9 , IC should be 4282985 . When the two factors (HR and QR) work together, HR plays a decisive role in the trend of the curve and determines the overall trend of the epidemic situation. By contrast, the growth of QR makes the number of infected people and deaths show a relatively small downward trend.

Figure 13(b) shows the values of KC under different HRs and QRs. Given a fixed value of QR, while HR is within the 
interval of $[0,0.5], \mathrm{KC}$ increases with HR. At this stage, the number of infected cases that can be accommodated in the hospitals is small, and most of the patients who died from infection are not included in the statistics. While HR is within the interval of $[0.5,1], \mathrm{KC}$ gradually decreases with $\mathrm{HR}$. Moreover, given a fixed value of $\mathrm{HR}, \mathrm{KC}$ decreases with the increase of QR. Specifically, while both $\mathrm{HR}$ and QR are $0.1, \mathrm{KC}$ is 10399 . By contrast, if QR increases to 0.9 , then KC decreases to 6307 correspondingly.

Therefore, the hospital admission rate is more important than the quarantine rate in responding to the epidemic. The increase of the quarantine rate can only delay the peak of the epidemic but cannot determine the scale of the epidemic. On the contrary, enhancing the hospital admission rate (or treatment rate) can radically restrain the epidemic.

4.2.6. Combined Impact of Average Contact Number and Contact Infection Rate. This section studies the values of IC and $\mathrm{KC}$ on the 30th day under different NNs and ERs. In this simulation, HR and QR are set as 0.9 and 0.94 , respectively. $\mathrm{NN}$ and ER were regarded as the experimental variables, while other variables were regarded as the control variables. The value of $\mathrm{NN}$ is set between 0 and 3, while ER is between 0.03 and 0.06. The simulation results are shown in Figure 14.

Figure 14(a) shows the values of IC under different NNs and ERs. Given a fixed value of NN, IC increases with ER. Specifically, while NN is 3 and ER drops from 0.057 to 0.03 , IC drops from more than 9000 to 300 . Additionally, given a fixed value of ER, IC increases with NN. Specifically, while $\mathrm{ER}$ is 0.057 and $\mathrm{NN}$ increases from 0.3 to 3 , IC increase from 23 to more than 9000 .

Figure 14(b) shows the values of KC under different NNs and ERs. At the initial stage of the outbreak, the residents do not take the necessary protective measures, resulting in a high ER. With the increase of NN, KC grows rapidly. After the residents make personal protection, ER decreases and the growth rate of KC slows down. Similarly, KC increases with $\mathrm{NN}$, and the growth rate slows down with the decrease of ER.

It implies the increase of average contact number and contact infection rate make the cumulative number of infections and the cumulative number of deaths increase exponentially. Therefore, in the early stage of the epidemic, if the residents can improve their awareness of self-prevention, strictly take scientific protective measures, and reduce outdoor activities, the spread of the epidemic can be restrained to a great extent.

\section{Conclusions and Suggestions}

This research establishes an improved SEIR-SD model for the COVID-19 epidemic. The real scenarios of the epidemic in Wuhan are simulated successfully by this model, which proves the effectiveness of the model. Afterward, the impact of hospital administration rate, quarantine rate, average contact number, and contact infection rate on the cumulative number of infections and deaths are analyzed by simulation. The results show that both the medical and administrative efforts, especially in the early stage of the epidemic, are significant in reducing the number of infections and shortening the epidemic period. In the medical aspect, the more stringent quarantine brings the earlier inflection point of the epidemic; more importantly, improving the treatment rate significantly reduces the scale of the epidemic. In the administrative aspect, enforcing individual protection and strict community closure can effectively cut off the transmission of the virus and curb the spread of the epidemic. In this regard, this research proposes the following suggestions:

(1) Based on the accurate judgment of the epidemic situation, the government should implement strict and effective community closure measures at the preliminary stage of the epidemic. Specifically, it is necessary to close factories and schools, stop collective activities or large-scale crowd assemblies, and take traffic restrictions in villages, communities, and other areas to reduce close contact among residents.

(2) The residents must improve their protection, such as wearing masks and keeping social distance in public places. The government should ensure an adequate supply of protective supplies and sterilize public places.

(3) The national or regional medical resources, including medical supplies and medical care personnel, should be allocated reasonably. Establishing temporary treatment centers for mild patients and expanding the capacity of the existing hospital for severe patients are important ways to realize the quarantine and treatment of the patients.

(4) Accurate tracking of the close contacts of confirmed cases and the suspected cases is of great importance. It is also necessary to mobilize the social forces and resources to establish quarantine places and conduct quarantine on suspected cases and close contacts to cut off the transmission chain of the virus fundamentally.

(5) In the early stage of the development of the epidemic, we should focus on ensuring the hospital admission rate. On this basis, we should quarantine mild infected cases, which not only reduces the occupation of important medical resources but also helps to restrain the epidemic to a certain extent.

\section{Data Availability}

No data were used to support this study.

\section{Conflicts of Interest}

The authors declare that they have no conflicts of interest.

\section{Acknowledgments}

This research was funded by the National Natural Science Foundation of China, grant nos. 72071212 and 71603284; the Natural Science Foundation of Hubei Province, China, grant 
no. 2020CFB518; Major Research Project of Philosophy and Social Sciences in Universities of Hubei Province, China, grant no. 21ZD014; the Fundamental Research Expenses for the Central Universities of China, grant no. 2722021BZ039.

\section{References}

[1] F. Zhang, L. Li, and H. Y. Xuan, "Survey of transmission models of infectious diseases," System Engineering-Theory \& Practice, vol. 31, no. 9, pp. 1736-1744, 2011.

[2] G.-M. Tang and Z.-X. Wu, "Fragility and robustness of selfsustained oscillations in an epidemiological model on smallworld networks," Chaos: An Interdisciplinary Journal of Nonlinear Science, vol. 29, no. 2, Article ID 023119, 2019.

[3] S. Eubank, H. Guclu, V. S. Anil Kumar et al., "Modelling disease outbreaks in realistic urban social networks," Nature, vol. 429, no. 6988, pp. 180-184, 2004.

[4] L. Hufnagel, D. Brockmann, and T. Geisel, "Forecast and control of epidemics in a globalized world," Proceedings of the National Academy of Sciences, vol. 101, no. 42, pp. 15124-15129, 2004.

[5] W. O. Kermack and A. G. Mckendrick, "Contributions to the mathematical theory of epidemics," Proceedings of the Royal Society of London, vol. 115, pp. 700-721, 1927.

[6] B. Jhun, M. Jo, and B. Kahng, "Simplicial SIS model in scalefree uniform hypergraph," Journal of Statistical Mechanics: Theory and Experiment, vol. 2019, no. 12, Article ID 123207, 2019.

[7] X. Mu, Q. Zhang, and L. Rong, "Optimal vaccination strategy for an SIRS model with imprecise parameters and Lévy noise," Journal of the Franklin Institute, vol. 356, no. 18, Article ID 11385, 2019.

[8] H. Inaba, "Age-structured homogeneous epidemic systems with application to the MSEIR epidemic model," Journal of Mathematical Biology, vol. 54, no. 1, pp. 101-146, 2007.

[9] J. W. Forrester, "Industrial dynamics: a breakthrough for decision makers," Harvard Business Review, vol. 4, pp. 37-66, 1958.

[10] E. R. G. T. R. Assunção, F. A. F. Ferreira, I. MeiduteKavaliauskienè, C. Zopounidis, L. F. Pereira, and R. J. C. Correia, "Rethinking urban sustainability using fuzzy cognitive mapping and system dynamics," The International Journal of Sustainable Development and World Ecology, vol. 27, no. 3, pp. 261-275, 2020.

[11] E. Ekinci, Y. Kazancoglu, and S. K. Mangla, "Using system dynamics to assess the environmental management of cement industry in streaming data context," The Science of the Total Environment, vol. 715, Article ID 136948, 2020.

[12] Y. Shi, "Stochastic dynamic model of SARS spreading," Chinese Science Bulletin, vol. 48, no. 13, pp. 1287-1292, 2003.

[13] D. R. Zeng, X. H. Cheng, and J. A. Huang, "Study on the mathematical model of SARS epidemic prediction and control-an improved method based on the infectious disease model," Chinese Journal of Safety Science, vol. 14, pp. 41-45, 2004.

[14] Q. Li, X. Guan, P. Wu et al., "Early transmission dynamics in Wuhan, China, of novel coronavirus-infected pneumonia," New England Journal of Medicine, vol. 382, pp. 1199-1207, 2020.

[15] B. Tang, X. Wang, Q. Li et al., "Estimation of the transmission risk of the 2019-nCoV and its implication for public health interventions," Journal of Clinical Medicine, vol. 9, no. 2, p. $462,2020$.

[16] S. Zhao, Q. Lin, J. Ran et al., "Preliminary estimation of the basic reproduction number of novel coronavirus (2019-
$\mathrm{nCoV}$ ) in China, from 2019 to 2020: a data-driven analysis in the early phase of the outbreak," International Journal of Infectious Diseases, vol. 92, pp. 214-217, 2020.

[17] Y. Y. Wei, Z. Z. Lu, and Z. C. Du, "Fitting and forecasting the trend of COVID-19 by SEIR+ CAQ dynamic model," Chinese Journal of Epidemiology, vol. 41, no. 04, pp. 470-475, 2020.

[18] J. M. Read, J. R. Bridgen, and D. A. Cummings, "Novel coronavirus 2019-nCoV: early estimation of epidemiological parameters and epidemic predictions," Philosophical Transactions of the Royal Society B: Biological Sciences, vol. 376, no. 1829, Article ID 20200265, 2020.

[19] M. W. Shen, Z. H. Peng, and Y. N. Xiao, "Modeling the epidemic trend of the 2019 novel coronavirus outbreak in China," The Innovation, vol. 1, no. 3, Article ID 100048, 2020.

[20] J. T. Wu, K. Leung, and G. M. Leung, "Nowcasting and forecasting the potential domestic and international spread of the 2019-nCoV outbreak originating in Wuhan, China: a modelling study," The Lancet, vol. 395, no. 10225, pp. 689-697, 2020.

[21] Q. Lin, S. Zhao, D. Gao et al., "A conceptual model for the coronavirus disease 2019 (COVID-19) outbreak in Wuhan, China with individual reaction and governmental action," International Journal of Infectious Diseases, vol. 93, pp. 211216, 2020.

[22] J. K. Adam, W. R. Timothy, and D. Charlie, "Early dynamics of transmission and control of COVID-19: a mathematical modeling study," The Lancet Infectious Diseases, vol. 20, no. 5, pp. 553-558, 2020.

[23] The Lancet, "COVID-19: too little, too late?" The Lancet, vol. 395 , no. 10226 , p. 755,2020 , https://www.sciencedirect. com/science/article/pii/S0140673620305225.

[24] S. He, Y. Peng, and K. Sun, "SEIR modeling of the COVID-19 and its dynamics," Nonlinear Dynamics, vol. 101, no. 3, pp. 1667-1680, 2020.

[25] S. Zhao and H. Chen, "Modeling the epidemic dynamics and control of COVID-19 outbreak in China," Quantitative Biology, vol. 8, no. 1, pp. 11-19, 2020.

[26] D. Kang, H. Choi, J.-H. Kim, and J. Choi, "Spatial epidemic dynamics of the COVID-19 outbreak in China," International Journal of Infectious Diseases, vol. 94, pp. 96-102, 2020.

[27] K. Linka, M. Peirlinck, F. Sahli Costabal, and E. Kuhl, "Outbreak dynamics of COVID-19 in Europe and the effect of travel restrictions," Computer Methods in Biomechanics and Biomedical Engineering, vol. 23, no. 11, pp. 710-717, 2020.

[28] J. S. Jia, X. Lu, and Y. Yuan, "Population flow drives spatiotemporal distribution of COVID-19 in China," Nature, vol. 582, no. 7812, 2020.

[29] G. Wei-jie, N. Zheng-yi, H. Yu, and L. Wenhua, "Clinical characteristics of 2019 novel coronavirus infection in China," New England Journal of Medicine, vol. 382, no. 18, pp. 1708-1720, 2020.

[30] M. Peirlinck, K. Linka, F. Sahli Costabal, and E. Kuhl, "Outbreak dynamics of COVID-19 in China and the United States," Biomechanics and Modeling in Mechanobiology, pp. 1-15, 2020.

[31] F. Zhou, T. Yu, R. Du et al., "Clinical course and risk factors for mortality of adult inpatients with COVID-19 in Wuhan, China: a retrospective cohort study," The Lancet, vol. 395, no. 10229, pp. 1054-1062, 2020.

[32] Wuhan Statistics Bureau, Wuhan Statistical Yearbook, Wuhan Publishing House, Wuhan, China, 2018.

[33] B. David, X. L. Qi, K. Nielsen-Saines, M Didier, P Léo, and F Guillaume, "Real estimates of mortality following COVID- 
19 infection," The Lancet Infectious Diseases, vol. 20, no. 7, p. $773,2020$.

[34] D. M. Studdert and M. A. Hall, "Disease control, civil liberties, and mass testing - calibrating restrictions during the covid-19 pandemic," New England Journal of Medicine, vol. 383, no. 2, pp. 102-104, 2020.

[35] M. Peirlinck, K. Linka, C. F. Sahli, and K Ellen, "Outbreak dynamics of COVID-19 in China and the United States," Biomechanics and Modeling in Mechanobiology, pp. 1-15, 2020.

[36] P. Neal and T. Theparod, "The basic reproduction number, R0, in structured populations," Mathematical Biosciences, vol. 315, p. 108224, 2019.

[37] Y. Chen, A. H. Wang, B. Yi, K. Q. Ding, H Wang, and J Wang, "The epidemiological characteristics of infection in close contacts of COVID-19 in Ningbo city," Chinese Journal of Epidemiology, vol. 41, no. 5, pp. 667-671, 2020. 